\title{
Asymptotics of linear differential systems and application to quasi-normal modes of nonrotating black holes
}

\author{
David Langlois, ${ }^{1}$ Karim Noui, ${ }^{2,1}$ and Hugo Roussille ${ }^{1,2}$ \\ ${ }^{1}$ Université de Paris, CNRS, Astroparticule et Cosmologie, F-75006 Paris, France \\ ${ }^{2}$ Institut Denis Poisson (UMR 7013), Université de Tours, \\ Université d'Orléans, Parc de Grandmont, 37200 Tours, France
}

(Dated: December 15, 2021)

\begin{abstract}
The traditional approach to perturbations of nonrotating black holes in General Relativity uses the reformulation of the equations of motion into a radial second-order Schrödinger-like equation, whose asymptotic solutions are elementary. Imposing specific boundary conditions at spatial infinity and near the horizon defines, in particular, the quasi-normal modes of black holes. For more complicated equations of motion, as encountered for instance in modified gravity models with different background solutions and/or additional degrees of freedom, we present a new approach that analyses directly the first-order differential system in its original form and extracts the asymptotic behaviour of perturbations, without resorting to a second-order reformulation. As a pedagogical illustration, we apply this treatment to the perturbations of Schwarzschild black holes and then show that the standard quasi-normal modes can be obtained numerically by solving this first-order system with a spectral method. This new approach paves the way for a generic treatment of the asymptotic behaviour of black hole perturbations and the identification of quasi-normal modes in theories of modified gravity.
\end{abstract}

\section{INTRODUCTION}

The oscillations of black holes $(\mathrm{BH})$ have been studied theoretically for several decades. Today, with the first observations of gravitational waves emitted by $\mathrm{BH}$ mergers, one can now hope to observe directly these oscillations via their GW signatures, especially in the ringdown phase of the signal when the post-merger black hole relaxes to a Kerr black hole, according to General Relativity. One of the major goals of future detections will be to check whether the observed oscillations coincide with the predictions based on General Relativity (see e.g. 1, 2]). This is also an ideal playground to test alternative theories of gravitation. Indeed, even if the background $\mathrm{BH}$ solution may coincide with that of GR, the linear perturbations in general obey different equations of motion.

During the ringdown phase, at least in the linear regime, the GW signal is expected to mainly consist of a superposition of the so-called quasi-normal, or resonant, modes (QNMs) which have been excited by the merger and then decay via GW radiation: these modes correspond to the proper oscillation modes of the black hole and are characterised by a complex frequency $\omega$, whose imaginary part quantifies their damping rate.

In the simplest case of nonrotating black holes, i.e. Schwarzschild black holes, the computation of QNMs is based on the classical papers by Regge \& Wheeler [3] and later Zerilli [4], who reformulated the linearised Einstein equations in the frequency domain, which are first-order with respect to the radial coordinate, as a second-order Schrödinger-like equation. This familiar equation, with a specific potential for axial and polar metric perturbations, is the standard starting point for the numerical calculations or semi-analytical treatments of QNMs, using for instance well-known methods in quantum mechanics.

Understanding the asymptotic behaviour of the perturbations at the horizon and at spatial infinity is crucial for QNMs, which are defined by very specific boundary conditions. Indeed, they correspond to purely outgoing radiation at spatial infinity and ingoing radiation at the horizon. Imposing these specific boundary conditions leads to a discrete set of allowed frequencies. 
When the equations of motion of the perturbations are written as a second-order Schrödinger equation, obtaining their asymptotic behaviour is immediate, as it simply depends on the asymptotic behaviour of the effective potential. In the context of modified gravity however, the problem can become more involved for several reasons. First, the background metric can differ from the standard GR solutions, i.e. be different from Schwarzschild in the nonrotating case. Moreover, modified theories often involve additional fields, such as scalar fields, which increases the number of degrees of freedom and therefore the complexity of the linear equations of motion.

In several interesting cases, the equations of motion can be rewritten as a generalised $N$ dimensional matrix Schrödinger-like system for $N$ fields $\Psi_{i}$, of the typical form (see e.g. [5])

$$
f \frac{d}{d r}\left(f \frac{d \Psi_{i}}{d r}\right)+\left(\omega^{2}-f V_{i j}\right) \Psi_{j}=0
$$

where $f(r)=1-r_{s} / r$ and the $N \times N$ matrix $V_{i j}$ of radial potentials usually vanishes or becomes a constant diagonal matrix asymptotically. The frequency $\omega$ appears quadratically in the above system, which corresponds to a system of propagation equations if one replaces $\omega$ with $-i \partial / \partial t$. The boundary conditions are still easy to infer from such a differential system.

However, one could also encounter more general situations where such a simple reformulation of the equations of motion is not available or would require an involved and lengthy procedure. Specific examples will be given in a companion paper [6], in the context of Degenerate Higher-Order Scalar-Tensor (DHOST) theories [7-10] which provide the most general viable set of scalar-tensor theories to date. In those examples, it is not clear whether one can rewrite the polar equations of motion as a second-order Schrödinger-like system of the form (1.1), with its specific dependence on $\omega$. In the specific case of stealth Schwarzschild black holes, a lengthy manipulation of the quadratic Lagrangian for perturbations enabled the authors of [11] to identify master variables, leading to a second-order differential system for the physical degrees of freedom, although of a more complex form than (1.1). To tackle more general situations, it would be very useful to be able to analyse directly the first-order system of equations in its original form and to extract directly from it the asymptotic behaviour of perturbations.

The purpose of this paper is to present such a systematic treatment of a general first-order differential system. In order to reach this goal, we use recent developments that appeared in the mathematical literature. These results enable us to determine, via a systematic algorithm, the asymptotic structure of the solutions of a generic first-order differential system. For pedagogical reasons, we use here this algorithm to recover the asymptotic solutions for the axial, or odd-parity, modes and for the polar, or even-parity, modes of the standard Schwarzschild solution. This paper will be completed by a companion paper [6] that applies the same method to a few black hole solutions in DHOST theories.

The outline of the paper is the following. In the next section, we review the standard derivation for the Schwarzschild perturbations, distinguishing as usual the axial and polar modes. In section III, we present our new approach and show explicitly how this new method enables us to recover the usual asymptotic solution, working directly with the first order system. We also show how the quasi-normal modes can be computed in this new perspective. We then present, in section IV, the general algorithm, carefully listing the various steps of the algorithm depending on the structure of the system. We give a summary and open some perspectives in the concluding section. A few appendices contain some additional details. 


\section{A SHORT REVIEW ON REGGE-WHEELER AND ZERILLI EQUATIONS}

In this section, we review the standard procedure to derive the equations of motion for the perturbations of a Schwarzschild black hole in general relativity, originally obtained by Regge and Wheeler [3] for the axial, or odd-parity, modes and Zerilli [4] for the polar, or even-parity, modes. These equations can be shown to reduce to a Schrödinger-like equation with an effective potential characterising the "dynamics" of the linear perturbations.

\section{A. Linear perturbations of Einstein equations about the Schwarzschild black hole}

We start with the four-dimensional Einstein-Hilbert action in vacuum (with no cosmological constant) for the metric $g_{\mu \nu}$,

$$
S\left[g_{\mu \nu}\right]=\frac{1}{16 \pi G_{N}} \int \mathrm{d}^{4} x \sqrt{-g} R
$$

where $g \equiv \operatorname{det}\left(g_{\mu \nu}\right)$ is the determinant of the metric, $R$ the four-dimensional Ricci scalar and $G_{N}$ denotes Newton's constant, which actually will not show up in the equations of motion since we are not considering any matter field here.

\section{Linearised general relativity}

Given any background metric $\bar{g}_{\mu \nu}$ solution to the Einstein equations, one can introduce the perturbed metric

$$
g_{\mu \nu}=\bar{g}_{\mu \nu}+h_{\mu \nu}
$$

where the $h_{\mu \nu}$ denote the linear perturbations of the metric. In order to derive the linear equations of motion that govern the evolution of $h_{\mu \nu}$, one expands the Einstein-Hilbert action (2.1) up to the second order in $h_{\mu \nu}$. The Euler-Lagrange equations associated with the quadratic part of this expansion then provide the linearised equations of motion for $h_{\mu \nu}$.

By expanding (2.1), one obtains the following quadratic action for $h_{\mu \nu}$,

$$
\begin{aligned}
S_{\text {quad }}\left[h_{\mu \nu}\right]=\frac{1}{16 \pi G_{N}} \int & \mathrm{d}^{4} x \sqrt{-\bar{g}}\left\{-\frac{1}{2} h_{\mu \nu} h^{\mu \nu} \bar{R}+\frac{1}{4} h^{2} \bar{R}+h h_{\mu \nu} \bar{R}^{\mu \nu}+4 h_{\mu}{ }^{\rho} h^{\mu \nu} \bar{R}_{\nu \rho}-2 h^{\mu \nu} h^{\rho \sigma} \bar{R}_{\mu \rho \nu \sigma}\right. \\
& +\frac{1}{2}\left(\bar{\nabla}_{\mu} h\right)\left(\bar{\nabla}^{\mu} h\right)-2\left(\bar{\nabla}_{\mu} h^{\mu}{ }_{\nu}\right)\left(\bar{\nabla}_{\rho} h_{\nu}{ }^{\rho}\right)-\left(\bar{\nabla}_{\mu} h\right)\left(\bar{\nabla}_{\nu} h^{\mu \nu}\right) \\
& \left.+3\left(\bar{\nabla}_{\nu} h_{\mu \rho}\right)\left(\bar{\nabla}^{\rho} h^{\mu \nu}\right)-\frac{1}{2}\left(\bar{\nabla}_{\rho} h_{\mu \nu}\right)\left(\bar{\nabla}^{\rho} h^{\mu \nu}\right)\right\}
\end{aligned}
$$

where $\bar{R}_{\mu \nu \rho \sigma}, \bar{R}_{\mu \nu}, \bar{R}$ and $\bar{\nabla}_{\mu}$ are respectively the Riemann tensor, the Ricci tensor, the Ricci scalar and the covariant derivative associated with the background metric $\bar{g}_{\mu \nu}$. The indices are lowered or raised with $\bar{g}_{\mu \nu}$ and $h \equiv \bar{g}^{\mu \nu} h_{\mu \nu}$ denotes the trace of the metric perturbation. The linearised Einstein equations are then given by the Euler-Lagrange equations of (2.3) and can be written in the form

$$
\begin{aligned}
\mathcal{E}_{\mu \nu} & \equiv \bar{\nabla}_{\sigma} \bar{\nabla}^{\sigma} h_{\mu \nu}+\bar{\nabla}_{\mu} \bar{\nabla}_{\nu} h+\left(\bar{\nabla}_{\alpha} \bar{\nabla}_{\beta} h^{\alpha \beta}-\bar{\nabla}_{\sigma} \bar{\nabla}^{\sigma} h\right) \bar{g}_{\mu \nu}+2 \bar{\nabla}_{(\mu} \bar{\nabla}_{\alpha} h_{\nu)}^{\alpha}-6 \bar{\nabla}_{\alpha} \bar{\nabla}_{(\mu} h_{\nu)}^{\alpha} \\
& +\bar{R}_{\mu \nu} h-\bar{R} h_{\mu \nu}+\frac{1}{2} \bar{R}_{\mu \nu} h+\bar{R}^{\alpha \beta} \bar{g}_{\mu \nu} h_{\alpha \beta}+8 \bar{R}_{\alpha(\mu} h_{\nu)}^{\alpha}=0
\end{aligned}
$$


where use the standard notation $A_{(\mu \nu)} \equiv\left(A_{\mu \nu}+A_{\nu \mu}\right) / 2$ for the symmetrisation of any rank-2 tensor $A_{\mu \nu}$.

Let us now specialise these equations to the case where the background metric is the Schwarzschild metric, expressed as

$$
\bar{g}_{\mu \nu} \mathrm{d} x^{\mu} \mathrm{d} x^{\nu}=-\left(1-\frac{r_{s}}{r}\right) \mathrm{d} t^{2}+\left(1-\frac{r_{s}}{r}\right)^{-1} \mathrm{~d} r^{2}+r^{2}\left(\mathrm{~d} \theta^{2}+\sin ^{2} \theta \mathrm{d} \varphi^{2}\right),
$$

where $r_{s}=2 M_{s}$ is the Schwarzschild radius, $M_{s}$ being the mass of the black hole.

Given the spherical symmetry of the background solution, it is convenient to decompose the metric perturbations $h_{\mu \nu}$ into (scalar, vectorial and tensorial) spherical harmonics that are defined from the standard $Y_{\ell m}(\theta, \varphi)$ functions and their derivatives with respect to $\theta$ and $\varphi$. They are labelled by the two multipole integers $\ell$ and $m$ (with $\ell \geq 0$ and $-\ell \leq m \leq \ell$ ).

Furthermore, one can distinguish axial and polar modes, which behave differently under the parity transformation $\vec{r} \rightarrow-\vec{r}$ : the polar, or even-parity, modes transform as $(-1)^{\ell}$, similarly to the scalar spherical harmonics $Y_{\ell m}(\theta, \varphi)$, whereas the axial, or odd-parity, modes transform as $(-1)^{\ell+1}$. These modes can be treated separately as they are decoupled at linear order. Moreover, we consider here only the modes $\ell \geq 2$. The particular cases of the $\ell=0$ and $\ell=1$ modes are briefly discussed in Appendix A3.

Since the background metric is static, it is also convenient to decompose the time dependence of the perturbations into Fourier modes,

$$
F(t, r)=\int_{-\infty}^{+\infty} \mathrm{d} \omega \tilde{F}(\omega, r) e^{-i \omega t}
$$

In the rest of this paper, we will use the same notation for the time-dependent function $F$ and its Fourier transform, as there will be no ambiguity. From a practical point of view, we simply replace every time derivative by a multiplication by $-i \omega$ in the linearised equations, which leads to a system of ordinary differential equations with respect to the radial variable $r$.

In both axial and polar sectors, the equations of motion can be reduced to a system of two first order ordinary differential equations, as we will show below.

\section{Axial perturbations}

We choose the usual Regge-Wheeler gauge [3 to describe the axial modes. As recalled in Appendix A 1, in this gauge the perturbations for $\ell \geq 2$ are parametrised by three families of functions $h_{0}^{\ell m}, h_{1}^{\ell m}$ and $h_{2}^{\ell m}$ according to

$$
\begin{array}{lll}
h_{t \theta}=\frac{1}{\sin \theta} \sum_{\ell, m} h_{0}^{\ell m} \partial_{\varphi} Y_{\ell m}(\theta, \varphi), & h_{t \varphi}=-\sin \theta \sum_{\ell, m} h_{0}^{\ell m} \partial_{\theta} Y_{\ell m}(\theta, \varphi), \\
h_{r \theta}=\frac{1}{\sin \theta} \sum_{\ell, m} h_{1}^{\ell m} \partial_{\varphi} Y_{\ell m}(\theta, \varphi), & h_{r \varphi}=-\sin \theta \sum_{\ell, m} h_{1}^{\ell m} \partial_{\theta} Y_{\ell m}(\theta, \varphi),
\end{array}
$$

while the other components vanish.

For these perturbations, the equations of motion (2.4) reduce to the following three equations

$$
\begin{aligned}
& \mathcal{E}_{t \theta}=2\left(\frac{r_{s}}{r}-1-\lambda\right) h_{0}(t, r)+r\left(r-r_{s}\right) \frac{\partial^{2} h_{0}}{\partial r^{2}}-2\left(r-r_{s}\right) \frac{\partial h_{1}}{\partial t}-r\left(r-r_{s}\right) \frac{\partial^{2} h_{1}}{\partial t \partial r}=0, \\
& \mathcal{E}_{r \theta}=-2 \lambda h_{1}(t, r)-\frac{2 r^{2}}{r-r_{s}} \frac{\partial h_{0}}{\partial t}+\frac{r^{3}}{r-r_{s}} \frac{\partial^{2} h_{0}}{\partial t \partial r}-\frac{r^{3}}{r-r_{s}} \frac{\partial^{2} h_{1}}{\partial t^{2}}=0, \\
& \mathcal{E}_{\theta \theta}=2 r_{s} h_{1}(t, r)+2 r\left(r-r_{s}\right) \frac{\partial h_{1}}{\partial r}-\frac{2 r^{3}}{r-r_{s}} \frac{\partial h_{0}}{\partial t}=0
\end{aligned}
$$


where we have introduced the notation

$$
2 \lambda \equiv \ell(\ell+1)-2
$$

as the equations $\mathcal{E}_{t \varphi}=0, \mathcal{E}_{r \varphi}=0, \mathcal{E}_{\varphi \varphi}=0$ and $\mathcal{E}_{\theta \varphi}=0$ are identical to the above ones.

Since there are only two independent functions, $h_{0}$ and $h_{1}$, one expects one of the above equations to be redundant. This is indeed verified by noting the following relation between the equations (2.8) and their derivatives, written now in the frequency domain,

$$
\frac{\mathrm{d} \mathcal{E}_{r \theta}}{\mathrm{d} r}+\frac{i r^{2} \omega}{\left(r-r_{s}\right)^{2}} \mathcal{E}_{t \theta}+\frac{r_{s}}{r\left(r-r_{s}\right)} \mathcal{E}_{r \theta}+\frac{\lambda}{r\left(r-r_{s}\right)} \mathcal{E}_{\theta \theta}=0
$$

This shows that the two equations $\mathcal{E}_{r \theta}=0$ and $\mathcal{E}_{\theta \theta}=0$ are sufficient to fully describe the dynamics of axial perturbations. As a consequence, the initial system 2.8 reduces to

$$
\frac{\mathrm{d} Y}{\mathrm{~d} r}=M(r) Y, \quad M(r)=\left(\begin{array}{cc}
2 / r & 2 i \lambda\left(r-r_{s}\right) / r^{3}-i \omega^{2} \\
-i r^{2} /\left(r-r_{s}\right)^{2} & -r_{s} / r\left(r-r_{s}\right)
\end{array}\right),
$$

where the two components of the column vector $Y \equiv{ }^{T}\left(Y_{1}, Y_{2}\right)$ are $Y_{1}(r) \equiv h_{0}(r)$ and $Y_{2}(r) \equiv$ $h_{1}(r) / \omega$. Notice that we divided the variable $h_{1}(r)$ by $\omega$ in the definition of $Y_{2}$ in order to get a system which does not involve powers of $\omega$ higher than 2 , or equivalently which is at most second order in time if one inverts the Fourier transform 2.6.

\section{Polar perturbations}

After fixing the gauge, polar perturbations are parametrised by four families of functions $H_{0}^{\ell m}, H_{1}^{\ell m}, H_{2}^{\ell m}$ and $K^{\ell m}$ as shown in Appendix A 2 . The nonvanishing metric perturbations then read

$$
\begin{array}{ll}
h_{t t}=A(r) \sum_{\ell, m} H_{0}^{\ell m}(t, r) Y_{\ell m}(\theta, \varphi), & h_{t r}=\sum_{\ell, m} H_{1}^{\ell m}(t, r) Y_{\ell m}(\theta, \varphi), \\
h_{r r}=\frac{1}{A(r)} \sum_{\ell, m} H_{2}^{\ell m}(t, r) Y_{\ell m}(\theta, \varphi), & h_{a b}=\sum_{\ell, m} K^{\ell m}(t, r) g_{a b} Y_{\ell m}(\theta, \varphi),
\end{array}
$$

where $A(r) \equiv 1-r_{s} / r$ is included in the definitions for later convenience, and the indices $a$ or $b$ in the last equation are the angles $\theta$ or $\varphi$.

The linearised Einstein's equations yield seven distinct equations, which can be found in (B1) of Appendix B. After a few manipulation, also discussed in Appendix B, one finds that these equations of motion can be reduced to two first-order equations only. In the frequency domain, they read

$$
\frac{\mathrm{d} Y}{\mathrm{~d} r}=M(r) Y, \quad M(r)=\frac{1}{3 r_{s}+2 \lambda r}\left(\begin{array}{cc}
\frac{r_{s}\left(3 r_{s}+(\lambda-2) r\right)-2 r^{4} \omega^{2}}{r\left(r-r_{s}\right)} & \frac{2 i(\lambda+1)\left(r_{s}+\lambda r\right)+2 i r^{3} \omega^{2}}{r^{2}} \\
\frac{i r\left(9 r_{s}^{2}-8 \lambda r^{2}+8(\lambda-1) r_{s} r\right)+4 i r^{5} \omega^{2}}{2\left(r-r_{s}\right)^{2}} & \frac{2 r^{4} \omega^{2}-r_{s}\left(3 r_{s}+3 \lambda r+r\right)}{r\left(r-r_{s}\right)}
\end{array}\right)
$$

where now the two components of $Y$ are defined by $Y_{1}(r) \equiv K(r)$ and $Y_{2}(r) \equiv H_{1}(r) / \omega$. Similarly to the axial sector, the definition of $Y_{2}$ is motivated by the fact that the resulting system involves at most $\omega^{2}$ terms. 


\section{B. Schrödinger-like equation and effective potential}

In both axial and polar sectors, the equations of motion have been recast in the form of a system consisting simply of two first-order differential equations (with respect to the radial variable), namely (2.11) for axial perturbations and (2.14) for polar perturbations. In both cases, we now recall how this system can be rewritten as a Schrödinger-like equation.

\section{From the first order system to the Schrödinger-like equation}

As shown in [3] and [4], one can rewrite these systems as a single second order (in radial derivatives) Schrödinger-like equation for a unique dynamical variable. Reformulating a first order system of this kind as a Schrödinger equation is, in general, not an easy task because one has to ensure that the Schrödinger equation is second order in time and in space. It requires, in particular, a decoupling of the dynamical variables involved in the original first order system and a "clever" choice for the dynamical variable that should satisfy the second order Schrödinger equation.

In this section, we will describe how this works for the two systems (2.11) and (2.14) which take the general form

$$
\frac{\mathrm{d} Y}{\mathrm{~d} r}=M(r) Y
$$

where the coefficients of the matrix $M$ are polynomials (of degree at most 2) in $\omega$ and rational functions in $r$.

First, we consider the general (linear) change of vector

$$
Y(r)=P(r) \hat{Y}(r),
$$

where $\hat{Y}$ is a new column vector and the two dimensional invertible matrix $P$ has not been fixed at this stage. We also define a new radial coordinate $r_{*}$ and introduce the "Jacobian" of the transformation $n(r) \equiv \mathrm{d} r / \mathrm{d} r_{*}$. Now, the idea is to show that it is possible to find a matrix $P$ such that the new system satisfied by $\hat{Y}$ takes the canonical form

$$
\frac{\mathrm{d} \hat{Y}}{\mathrm{~d} r_{*}}=\left(\begin{array}{cc}
0 & 1 \\
V(r)-\omega^{2} & 0
\end{array}\right) \hat{Y}
$$

where the potential $V(r)$ depends on $r$, but not on $\omega$. Somehow, the first component $\hat{Y}_{1}$ plays the role of the "momentum" conjugate to the second component $\hat{Y}_{2}$ which would immediately implies that $\hat{Y}_{1}$ is the "canonical" variable satisfying the required Schrödinger-like equation

$$
\frac{\mathrm{d}^{2} \hat{Y}_{1}}{\mathrm{~d} r_{*}^{2}}+\left(\omega^{2}-V(r)\right) \hat{Y}_{1}=0
$$




\section{Axial modes}

Applying this procedure to the system 2.11) for the axial perturbations is rather simple ${ }^{1}$. Indeed, the appropriate transition matrix is given by

$$
P(r)=\left(\begin{array}{cc}
1-r_{s} / r & r \\
-i r^{2} /\left(r-r_{s}\right) & 0
\end{array}\right)
$$

while $n(r)=1-r_{s} / r$, which means that $r_{*}$ coincides with the "tortoise" coordinate,

$$
r_{*} \equiv \int \frac{d r}{1-r_{s} / r}=r+r_{s} \ln \left(r / r_{s}-1\right)
$$

Finally the effective potential $V_{\text {odd }}(r)$ for the axial perturbations takes the form

$$
V_{\text {odd }}(r)=\left(1-\frac{r_{s}}{r}\right) \frac{2(\lambda+1) r-3 r_{s}}{r^{3}} .
$$

Note that this potential vanishes both at spatial infinity $(r \rightarrow+\infty)$ and at the horizon $\left(r \rightarrow r_{s}\right)$.

\section{Polar modes}

The case of polar perturbations is slightly more involved. Starting from the system (2.14), we find that the transition matrix leading to a canonical form 2.17 is given by ${ }^{2}$

$$
P=\left(\begin{array}{cc}
\frac{3 r_{s}^{2}+3 \lambda r_{s} r+2 r^{2} \lambda(\lambda+1)}{2 r^{2}\left(3 r_{s}+2 \lambda r\right)} & 1 \\
-i+\frac{i r_{s}}{2\left(r-r_{s}\right)}+\frac{3 i r_{s}}{3 r_{s}+2 \lambda r} & -\frac{i r^{2}}{r-r_{s}}
\end{array}\right),
$$

with, in addition, $n(r)=1-r_{s} / r$, which means that $r_{*}$ is still the tortoise coordinate (2.21). Finally, the corresponding potential $V_{\text {even }}(r)$ reads

$$
V_{\text {even }}(r)=\left(1-\frac{r_{s}}{r}\right) \frac{9 r_{s}^{3}+18 r_{s}^{2} r \lambda+12 r_{s} r^{2} \lambda^{2}+8 r^{3} \lambda^{2}(1+\lambda)}{r^{3}\left(3 r_{s}+2 r \lambda\right)^{2}}
$$

${ }^{1}$ When one changes variables according to 2.16 , the new variable $\hat{Y}$ satisfies the differential equation

$$
\frac{\mathrm{d} \hat{Y}}{\mathrm{~d} r_{*}}=\hat{M} \hat{Y}, \quad \hat{M} \equiv n(r)\left(P^{-1} M P-P^{-1} P^{\prime}\right),
$$

where $P^{\prime}$ is the derivative of $P$ with respect to $r, M$ is the matrix introduced in 2.11 while $\hat{M}$ is the matrix entering in the system 2.17). They take a similar form $M=M_{[0]}+\omega^{2} M_{[2]}$ and $M=\hat{M}_{[0]}+\omega^{2} \hat{M}_{[2]}$ where the expressions of $M_{[0]}, M_{[2]}, \hat{M}_{[0]}$ and $\hat{M}_{[2]}$ are trivially obtained. As $P$ does not depend on $\omega$, the relation between $M$ and $\hat{M}$ translates into the two matricial relations $\hat{M}_{[2]}=n(r) P^{-1} M_{[2]} P$ and $\hat{M}_{[0]}=n(r)\left(P^{-1} M_{[0]} P-P^{-1} P^{\prime}\right)$ which can be viewed as 8 equations for the 6 unknowns $n(r), V(r)$ together with the four components of $P$. Interestingly, the system is not overdetermined and admits a solution for $P(2.20)$, for the potential $V(r) 2.22$, and for the function $n(r)$ which can be shown to be associated with the tortoise coordinate 2.21]. Details can be found in the Appendix D of the companion paper.

${ }^{2}$ We follow the same method as the one described in the previous footnote for the axial mode. 




FIG. 1 Illustration of the effective potentials (for axial and polar modes) for a Schwarzschild black hole. The parameters are such that $r_{s}=2$ (i.e. the mass of the black is 1 in natural units) and $\ell=2$ here.

Despite their different analytic forms, we notice in Fig.(1) that the potentials $V_{\text {odd }}(r)$ and $V_{\text {even }}(r)$ are quite similar, although distinct. In fact, there exists an underlying symmetry between these two potentials, further explained in [12], leading to the isospectrality theorem which states that the spectra of axial and polar perturbations are exactly the same.

\section{Quasi-normal modes and boundary conditions}

Finding quasi-normal modes requires to impose the appropriate boundary conditions: the modes must be outgoing at infinity and ingoing at the horizon.

Since both $V_{\text {odd }}$ and $V_{\text {even }}$ go to zero at infinity and at the horizon, equation (2.18) becomes asymptotically

$$
\frac{\mathrm{d}^{2} \hat{X}_{1}}{\mathrm{~d} r_{*}^{2}}+\omega^{2} \hat{X}_{1} \approx 0 \quad\left(r_{*} \rightarrow \pm \infty\right)
$$

where $\approx$ is an equality up to sub-leading corrections ${ }^{3}$. Therefore, at both boundaries, the function $\hat{X}_{1}$ behaves like

$$
\hat{X}_{1}(r) \approx \mathcal{A} e^{i \omega r_{*}}+\mathcal{B} e^{-i \omega r_{*}}
$$

where $\mathcal{A}$ and $\mathcal{B}$ are integration constants which take different values at the horizon and at infinity.

The physical interpretation of these modes is more transparent if we include their time dependence explicitly, which gives

$$
\left\{\begin{array}{l}
\hat{X}_{1}(t, r) \approx \mathcal{A}_{\text {hor }} e^{-i \omega\left(t-r_{*}\right)}+\mathcal{B}_{\text {hor }} e^{-i \omega\left(t+r_{*}\right)} \quad \text { when } r \longrightarrow r_{s}, \\
\hat{X}_{1}(t, r) \approx \mathcal{A}_{\infty} e^{-i \omega\left(t-r_{*}\right)}+\mathcal{B}_{\infty} e^{-i \omega\left(t+r_{*}\right)} \quad \text { when } r \longrightarrow \infty
\end{array}\right.
$$

We can interpret each term as a radially propagating wave: the terms proportional to $\mathcal{A}_{\text {hor }}$ and $\mathcal{A}_{\infty}$ are outgoing while the terms proportional to $\mathcal{B}_{\text {hor }}$ and $\mathcal{B}_{\infty}$ are ingoing. Imposing a purely outgoing behaviour at infinity and a purely ingoing behaviour at the horizon, i.e. such that $\mathcal{A}_{\text {hor }}=0$ and

\footnotetext{
${ }^{3}$ Near the horizon, $V=\mathcal{O}\left(r-r_{s}\right)$ for both potentials, hence we assume $r_{s}^{2} \omega^{2} \gg r / r_{s}-1$. At infinity, $V=\mathcal{O}\left(1 / r^{2}\right)$ for both potentials as well, hence we assume $\omega^{2} r^{2} \gg 1$ in this limit.
} 
$\mathcal{B}_{\infty}=0$ severely restricts the possible values of $\omega$. These values can be found numerically by integrating the Schrödinger-like equation (see [13] and the reviews [14 17]).

Finally, one can easily deduce the asymptotic expansion of the original gravitational perturbations using the transformations (2.16). For the axial modes, the leading order terms at infinity are thus given by

$$
h_{0}(r) \approx i \omega r\left(\mathcal{A}_{\infty} e^{i \omega r_{*}}-\mathcal{B}_{\infty} e^{-i \omega r_{*}}\right), \quad h_{1}(r) \approx-i \omega r\left(\mathcal{A}_{\infty} e^{i \omega r_{*}}+\mathcal{B}_{\infty} e^{-i \omega r_{*}}\right),
$$

while the leading order terms at the horizon read

$$
h_{0}(r) \approx i \omega r_{s}\left(\mathcal{A}_{\mathrm{hor}} e^{i \omega r_{*}}-\mathcal{B}_{\mathrm{hor}} e^{-i \omega r_{*}}\right), \quad h_{1}(r) \approx-\frac{i \omega r_{s}^{2}}{\varepsilon}\left(\mathcal{A}_{\mathrm{hor}} e^{i \omega r_{*}}+\mathcal{B}_{\mathrm{hor}} e^{-i \omega r_{*}}\right),
$$

where we have introduced the variable $\varepsilon \equiv r-r_{s}$ which satisfies $\varepsilon \ll r_{s}$ near the horizon.

For the polar modes, the leading order terms at infinity are

$$
K(r) \approx i \omega\left(\mathcal{A}_{\infty} e^{i \omega r_{*}}-\mathcal{B}_{\infty} e^{-i \omega r_{*}}\right), \quad H_{1}(r) \approx r \omega^{2}\left(\mathcal{A}_{\infty} e^{i \omega r_{*}}-\mathcal{B}_{\infty} e^{-i \omega r_{*}}\right)
$$

while the leading terms at the horizon are a bit more involved and read

$$
\begin{aligned}
K(r) & \approx \frac{\lambda+1+2 i \omega r_{s}}{r_{s}} \mathcal{A}_{\mathrm{hor}} e^{i \omega r_{*}}+\frac{\lambda+1-2 i \omega r_{s}}{r_{s}} \mathcal{B}_{\mathrm{hor}} e^{-i \omega r_{*}}, \\
H_{1}(r) & \approx \frac{i r_{s} \omega\left(1-2 i \omega r_{s}\right)}{2 \varepsilon} \mathcal{A}_{\mathrm{hor}} e^{i \omega r_{*}}+\frac{i r_{s} \omega\left(1+2 i \omega r_{s}\right)}{2 \varepsilon} \mathcal{B}_{\mathrm{hor}} e^{-i \omega r_{*}} .
\end{aligned}
$$

In the next section, we will recover these asymptotic behaviours in a completely different way.

\section{FIRST ORDER APPROACH TO SCHWARZSCHILD PERTURBATIONS}

As we have seen in section II B, finding a (second-order) Schrödinger-like equation for the metric perturbations starting from the Einstein equations requires some manipulations of the equations of motion and an appropriate choice of the function that verifies the Schrödinger-like equation.

The rest of this paper will be devoted to obtaining the asymptotic behaviours of the perturbations by using a different method. Although this is of course not necessary for the perturbations of Schwarzschild in General Relativity, our method may prove to be very useful in situations where a Schrödinger-like system is not obvious to find or even impossible to reach. In such a case, one would need an alternative method to determine the asymptotic limits of the solutions of the system, and from them, to compute the quasi-normal modes.

The general method will be described in a systematic way in the next section. As the general procedure is somewhat tedious, we have preferred to present it first, in a pedestrian way, for the perturbations of Schwarzschild. A more mathematically-minded reader might prefer to jump directly to the next section and later come back to this section to find a particular application of the general method.

\section{A. Method}

Ignoring the traditional Schrödinger reformulation, we now go back to the original first-order system given in (2.11) or 2.14). Schematically, we thus have a first-order system of the form

$$
\frac{\mathrm{d} Y}{\mathrm{~d} r}=M(r) Y
$$


where $Y(r)$ is a column vector and $M(r)$ a square matrix. In order to study the system at spatial infinity, say, i.e. when $r \rightarrow \infty$, one can expand the matrix $M(r)$ in powers of $r$,

$$
M(r)=M_{p} r^{p}+\cdots+M_{0}+M_{-1} \frac{1}{r}+\mathcal{O}\left(\frac{1}{r^{2}}\right)
$$

where all the matrix coefficients $M_{i}$ are $r$-independent. We stop here the expansion at order $1 / r$, which is sufficient for the simplest cases, but higher orders might be needed in general.

If all matrices $M_{i}$ are diagonal, it is immediate to integrate the truncated system, which then consists of $n$ ordinary differential equations of the form

$$
y^{\prime}(r)=\left(\lambda_{p} r^{p}+\cdots+\lambda_{0}+\frac{\mu}{r}\right) y(r)
$$

whose solution is

$$
y(r)=y_{0} e^{q(r)} r^{\mu}, \quad q(r)=\frac{\lambda_{p}}{p+1} r^{p+1}+\cdots+\lambda_{0} r .
$$

Putting together these $n$ solutions, we thus get the solution to the system (3.1), assuming all matrices $M_{i}$ in $(3.2)$ are diagonal, in the form

$$
Y(r)=e^{\boldsymbol{\Upsilon}(r)} r^{\boldsymbol{\Delta}} \mathbf{F}(r) Y_{0}
$$

where $Y_{0}$ is a constant vector, corresponding to the $n$ integration constants, $\boldsymbol{\Upsilon}$ is a diagonal matrix whose coefficients are polynomials of degree at most $p+1, \boldsymbol{\Delta}$ is a constant diagonal matrix and $\mathbf{F}(r)$ is a matrix which is regular at infinity (i.e. whose limit is finite).

Of course, in general, the matrices $M_{i}$ are not diagonal but, remarkably, it is always possible to transform the truncated system into a fully diagonal system, in a finite number of steps following an algorithm introduced in [18 21], which we will present in full details in the next section.

At each step in the algorithm, one introduces a new vector $\tilde{Y}$, related to the vector $Y$ of the previous step by

$$
Y=P \tilde{Y}
$$

where $P$ is an invertible matrix so that the previous system (3.1) is transformed into a new, but equivalent, system of the form

$$
\frac{\mathrm{d} \tilde{Y}}{\mathrm{~d} r}=\tilde{M}(r) \tilde{Y}, \quad \tilde{M}(r) \equiv P^{-1} M P-P^{-1} \frac{\mathrm{d} P}{\mathrm{~d} r} .
$$

The idea is then to choose an appropriate transition matrix $P$ at each step in order to diagonalise, order by order, the matrices that appear in the expansion of $M$. Once all the matrices are diagonalised, one can integrate directly the diagonal system, as we have seen earlier, and obtain the general asymptotic solution of the system.

For the asymptotic behaviour near the horizon, one proceeds in the same way by noting that the variable $z=1 /\left(r-r_{s}\right)$ goes to infinity when $r \rightarrow r_{s}$. In the rest of this section, we will illustrate the algorithm by considering in turn the asymptotic behaviours of the axial and polar modes.

\section{B. Axial modes}

The analysis of the asymptotic behaviour of the first order system (2.11) is relatively simple and instructive. We recall that the system is of the form

$$
\frac{\mathrm{d} Y}{\mathrm{~d} r}=M(r) Y,
$$


with

$$
Y(r) \equiv\left(\begin{array}{c}
h_{0}(r) \\
h_{1}(r) / \omega
\end{array}\right), \quad M(r) \equiv\left(\begin{array}{cc}
2 / r & 2 i \lambda\left(r-r_{s}\right) / r^{3}-i \omega^{2} \\
-i r^{2} /\left(r-r_{s}\right)^{2} & -r_{s} / r\left(r-r_{s}\right)
\end{array}\right)
$$

\section{Asymptotic analysis at spatial infinity}

We first study the asymptotic behaviour at spatial infinity, i.e. when $r \rightarrow \infty$. The asymptotic expansion of the matrix $M(r)$ at large $r$ reads

$$
M(r)=M_{0}+\frac{1}{r} M_{-1}+\mathcal{O}\left(\frac{1}{r^{2}}\right), \quad M_{0} \equiv-i\left(\begin{array}{cc}
0 & \omega^{2} \\
1 & 0
\end{array}\right), \quad M_{-1} \equiv 2\left(\begin{array}{cc}
1 & 0 \\
-i r_{s} & 0
\end{array}\right) .
$$

The leading term $M_{0}$ is diagonalisable and one can go to a basis where it is diagonal, by introducing the new vector $Y^{(1)}$ defined by

$$
Y \equiv P_{(1)} Y^{(1)}, \quad P_{(1)}=\left(\begin{array}{cc}
\omega & -\omega \\
1 & 1
\end{array}\right)
$$

According to (3.6), this gives the new system

$$
\frac{\mathrm{d} Y^{(1)}}{\mathrm{d} r}=M^{(1)} Y^{(1)}, \quad M^{(1)}(r)=M_{0}^{(1)}+\frac{1}{r} M_{-1}^{(1)}+\mathcal{O}\left(\frac{1}{r^{2}}\right),
$$

with

$$
M_{0}^{(1)} \equiv\left(\begin{array}{cc}
-i \omega & 0 \\
0 & i \omega
\end{array}\right), \quad M_{-1}^{(1)} \equiv\left(\begin{array}{cc}
-i \omega r_{s}+1 & i \omega r_{s}-1 \\
-i \omega r_{s}-1 & i \omega r_{s}+1
\end{array}\right)
$$

We need some extra work to diagonalise the next-to-leading order matrix $M_{-1}^{(1)}$ while keeping the leading order matrix diagonal.

This can be achieved by introducing a new vector $Y^{(2)}$ defined by

$$
Y^{(1)} \equiv P_{(2)} Y^{(2)}, \quad P_{(2)}=I+\frac{1}{r} \Xi,
$$

where $I$ is the identity matrix and $\Xi$ a constant matrix. Indeed, it is immediate to see that such a change of variable leads to the equivalent differential system,

$$
\frac{\mathrm{d} Y^{(2)}}{\mathrm{d} r}=M^{(2)} Y^{(2)}, \quad M^{(2)}(r)=M_{0}^{(2)}+\frac{1}{r} M_{-1}^{(2)}+\mathcal{O}\left(\frac{1}{r^{2}}\right),
$$

with

$$
M_{0}^{(2)}=M_{0}^{(1)}, \quad M_{-1}^{(2)}=M_{-1}^{(1)}+\left[M_{0}^{(1)}, \Xi\right]
$$

The leading matrix remains unchanged while one can easily find a matrix $\Xi$ so that $M_{-1}^{(2)}$ is diagonal. Notice that $\Xi$ appears in 3.15 only in a commutator with the diagonal matrix $M_{0}^{(1)}$, hence the diagonal part of $\Xi$ is irrelevant and we can already fix the diagonal terms of $\Xi$ to 0 . In this case, the solution to 3.15 with $M_{-1}^{(2)}$ diagonal is unique and given by

$$
\Xi=\frac{1}{2 i \omega}\left(\begin{array}{cc}
0 & i \omega r_{s}-1 \\
i \omega r_{s}+1 & 0
\end{array}\right)
$$


We have thus managed to obtain a fully diagonalised system, up to order $1 / r$, with the matrix

$$
M^{(2)}(r)=\left(\begin{array}{cc}
-i \omega & 0 \\
0 & i \omega
\end{array}\right)+\frac{1}{r}\left(\begin{array}{cc}
1-i \omega r_{s} & 0 \\
0 & 1+i \omega r_{s}
\end{array}\right)+\mathcal{O}\left(\frac{1}{r^{2}}\right)
$$

This system can be immediately integrated in the form (3.5), and the asymptotic solution reads

$$
Y^{(2)}(r)=(1+\mathcal{O}(1 / r))\left(\begin{array}{l}
c_{-} e^{-i \omega r} r^{1-i \omega r_{s}} \\
c_{+} e^{+i \omega r} r^{1+i \omega r_{s}}
\end{array}\right)
$$

where $c_{ \pm}$are integration constants. Taking into account the time dependency $e^{-i \omega t}$ of the modes, the two components $Y_{\mp}^{(2)}$ of $Y^{(2)}$ are of the form

$$
e^{-i \omega t} Y_{\mp}^{(2)}(r)=(1+\mathcal{O}(1 / r)) c_{\mp} r e^{-i \omega\left(t \pm\left(r+r_{s} \ln r\right)\right)}=c_{\mp}(r+\mathcal{O}(1)) e^{-i \omega\left(t \pm r_{*}\right)},
$$

where it is convenient to use the "tortoise" coordinate $r_{*}$, introduced in (2.21), noting that

$$
r_{*}=r+r_{s} \ln \left(r / r_{s}-1\right)=r+r_{s} \ln r+\mathcal{O}(1) .
$$

As a consequence, one can identify $Y_{-}^{(2)}$ as an ingoing mode and $Y_{+}^{(2)}$ as an outgoing mode at spatial infinity.

Finally, we can return to the original vector $Y$ thanks to the transformation

$$
Y=P_{(1)} P_{(2)} Y^{(2)}=\left(\begin{array}{cc}
\omega & -\omega \\
1 & 1
\end{array}\right)\left(1+\frac{\Xi}{r}\right) Y^{(2)}
$$

in order to obtain the asymptotic expansion of the two original gravitational perturbations $h_{0}$ and $h_{1}$ at spatial infinity,

$$
\begin{aligned}
& h_{0}(r)=\omega\left(c_{-} e^{-i \omega r_{*}}-c_{+} e^{+i \omega r_{*}}\right)(r+\mathcal{O}(1)), \\
& h_{1}(r)=\omega\left(c_{-} e^{-i \omega r_{*}}+c_{+} e^{+i \omega r_{*}}\right)(r+\mathcal{O}(1)) .
\end{aligned}
$$

One can immediately check that these expressions agree with the asymptotic expansion (2.28) obtained from the Schrödinger-like equation (with $c_{-}=-i \mathcal{B}_{\infty}$ and $c_{+}=-i \mathcal{A}_{\infty}$ ).

\section{Asymptotic analysis near the black hole horizon}

Let us now study the behaviour of the axial modes near the horizon. In this case, it is convenient to introduce the new radial variable $\varepsilon \equiv r-r_{s}$ and expand the matrix $M$ for the system (3.8) in powers of $\varepsilon$. One finds ${ }^{4}$

$$
M(\varepsilon)=\frac{1}{\varepsilon^{2}} M_{2}+\frac{1}{\varepsilon} M_{1}+M_{0}+\mathcal{O}(\varepsilon)
$$

\footnotetext{
${ }^{4}$ Note that $\varepsilon$ goes to zero here, in contrast to the previous case where the variable $r$ was going to infinity. One could work in a fully analogous system by using the variable $z=1 / \varepsilon$, with the system

$$
\frac{d Y}{d z}=\tilde{M}(z) Y, \quad \tilde{M}=-\frac{1}{z^{2}} M\left(z^{-1}\right)=-M_{2}-M_{1} \frac{1}{z}-M_{0} \frac{1}{z^{2}} .
$$

In the present case, one must push the expansion up to order $1 / z^{2}$ because the leading matrix $M_{2}$ is nilpotent. 
with the matrix coefficients

$$
M_{2} \equiv\left(\begin{array}{cc}
0 & 0 \\
-i r_{s}^{2} & 0
\end{array}\right), \quad M_{1} \equiv\left(\begin{array}{cc}
0 & 0 \\
-2 i r_{s} & -1
\end{array}\right), \quad M_{0} \equiv\left(\begin{array}{cc}
2 / r_{s} & -i \omega^{2} \\
-i & 1 / r_{s}
\end{array}\right)
$$

An important difference with the previous situation is that the leading term $M_{2}$ is no longer diagonalisable but nilpotent instead. We thus need to first perform a transformation that yields a diagonalisable leading matrix, taking advantage of the derivative term in (3.6). This can be done with the transformation

$$
Y \equiv P_{(1)} Y^{(1)}, \quad P_{(1)}(\varepsilon) \equiv\left(\begin{array}{cc}
1 & 0 \\
0 & 1 / \varepsilon
\end{array}\right)
$$

leading to the new system

$$
\frac{\mathrm{d} Y^{(1)}}{\mathrm{d} \varepsilon}=M^{(1)} Y^{(1)}, \quad M^{(1)}(\varepsilon)=-\frac{1}{\varepsilon}\left(\begin{array}{cc}
0 & i \omega^{2} \\
i r_{s}^{2} & 0
\end{array}\right)+\mathcal{O}(1) .
$$

The transformation (3.27) has eliminated the term in $1 / \varepsilon^{2}$ in the expansion and the leading term $M_{1}^{(1)}$ is now diagonalisable, so that only the expansion of $M^{(1)}$ up to order $1 / \varepsilon$ is required (see discussion in the footnote). It is worth noticing that $M_{1}^{(1)}$ receives contributions from $M_{2}, M_{1}$ and $M_{0}$. In particular, some of its coefficients involve the frequency $\omega$ which is originally present only in $M_{0}$.

The final step of the analysis consists in diagonalising the system (3.28), via the transformation

$$
Y^{(1)}=P_{(2)} Y^{(2)}, \quad P_{(2)} \equiv\left(\begin{array}{cc}
\omega & -\omega \\
r_{s} & r_{s}
\end{array}\right)
$$

leading to

$$
\frac{\mathrm{d} Y^{(2)}}{\mathrm{d} \varepsilon}=M^{(2)} Y^{(2)}, \quad M^{(2)}(\varepsilon) \equiv \frac{1}{\varepsilon}\left(\begin{array}{cc}
-i \omega r_{s} & 0 \\
0 & i \omega r_{s}
\end{array}\right)+\mathcal{O}(1)
$$

Integrating this equation yields

$$
Y^{(2)}(\varepsilon)=(1+\mathcal{O}(\varepsilon))\left(\begin{array}{c}
c_{-} \varepsilon^{-i \omega r_{s}} \\
c_{+} \varepsilon^{+i \omega r_{s}}
\end{array}\right)=(1+\mathcal{O}(\varepsilon))\left(\begin{array}{c}
c_{-} e^{-i \omega r_{*}} \\
c_{+} e^{+i \omega r_{*}}
\end{array}\right)
$$

where we have again expressed the result in terms of the tortoise coordinate $r_{*}$, which behaves as $r_{*}=r_{s} \ln \varepsilon+\mathcal{O}(1)$ near the horizon. One can immediately recognize the ingoing and outgoing modes at the horizon.

Finally, one can return to the original functions, via $Y=P_{(1)} P_{(2)} Y^{(2)}$, and derive the expressions

$$
\begin{aligned}
& h_{0}(r)=\omega\left(c_{-} e^{-i \omega r_{*}}-c_{+} e^{+i \omega r_{*}}\right)(1+\mathcal{O}(\varepsilon)), \\
& h_{1}(r)=\frac{\omega r_{s}}{\varepsilon}\left(c_{-} e^{-i \omega r_{*}}+c_{+} e^{+i \omega r_{*}}\right)(1+\mathcal{O}(\varepsilon)),
\end{aligned}
$$

which coincide with the asymptotic expansions 2.29) obtained from the Schrödinger-like equation (with $c_{-}=-i r_{s} \mathcal{B}_{\text {hor }}, c_{+}=-i r_{s} \mathcal{A}_{\text {hor }}$ ). 


\section{Polar modes} form

The dynamics of the polar perturbations is described by the first-order system (2.14), of the

$$
\frac{\mathrm{d} Y}{\mathrm{~d} r}=M(r) Y, \quad \text { with } \quad Y(r) \equiv\left(\begin{array}{c}
K(r) \\
H_{1}(r) / \omega
\end{array}\right)
$$

and the matrix

$$
M(r)=\frac{1}{3 r_{s}+2 \lambda r}\left(\begin{array}{cc}
\frac{r_{s}\left(3 r_{s}+(\lambda-2) r\right)-2 r^{4} \omega^{2}}{r\left(r-r_{s}\right)} & \frac{2 i(\lambda+1)\left(r_{s}+\lambda r\right)+2 i r^{3} \omega^{2}}{r^{2}} \\
\frac{i r\left(9 r_{s}^{2}-8 \lambda r^{2}+8(\lambda-1) r_{s} r\right)+4 i r^{5} \omega^{2}}{2\left(r-r_{s}\right)^{2}} & \frac{2 r^{4} \omega^{2}-r_{s}\left(3 r_{s}+3 \lambda r+r\right)}{r\left(r-r_{s}\right)}
\end{array}\right) .
$$

\section{Asymptotic analysis at spatial infinity}

Expanding 3.35 in powers of $r$, one gets

$$
\begin{aligned}
M(r) & =\left(\begin{array}{cc}
0 & 0 \\
\frac{i \omega^{2}}{\lambda} & 0
\end{array}\right) r^{2}+\left(\begin{array}{cc}
-\frac{\omega^{2}}{\lambda} & 0 \\
\frac{i r_{s} \omega^{2}(4 \lambda-3)}{2 \lambda^{2}} & \frac{\omega^{2}}{\lambda}
\end{array}\right) r+\left(\begin{array}{cc}
-\frac{(2 \lambda-3) r_{s} \omega^{2}}{2 \lambda^{2}} & \frac{i \omega^{2}}{\lambda} \\
-2 i+\frac{3 i\left(4 \lambda^{2}-4 \lambda+3\right) r_{s}^{2} \omega^{2}}{4 \lambda^{3}} & \frac{(2 \lambda-3) r_{s} \omega^{2}}{2 \lambda^{2}}
\end{array}\right) \\
& +\frac{1}{r}\left(\begin{array}{c}
-\frac{\left(4 \lambda^{2}-6 \lambda+9\right) r_{s}^{2} \omega^{2}}{\left.\left.\left.4 \lambda^{3}(2 x-9)+9\right)\right) r_{s}^{3} \omega^{2}\right)} \\
\frac{\left(4 \lambda^{2}-6 \lambda+9\right) r_{s}^{2} \omega^{2}}{4 \lambda^{3}}
\end{array}\right)+\mathcal{O}\left(\frac{1}{r^{2}}\right) .
\end{aligned}
$$

In contrast with the axial modes at spatial infinity, the leading matrix is of order $r^{2}$ and is nilpotent. So, in principle, one needs to apply a procedure similar to the near-horizon analysis of axial modes, which will be presented in full generality in the next section, and then diagonalise in turn all subsequent orders. All this involves many steps which are straightforward but rather tedious to describe.

To shorten our discussion, we provide directly the transformation that combines all these intermediate steps, given by

$$
Y=P \tilde{Y}, \quad P=\left(\begin{array}{ll}
\mathcal{S}+\mathcal{T} & \mathcal{S}-\mathcal{T} \\
\mathcal{U}-\mathcal{V} & \mathcal{U}+\mathcal{V}
\end{array}\right)
$$

with the functions

$$
\begin{aligned}
& \mathcal{S}(r) \equiv \frac{i\left(r-r_{s}\right)\left((2 \lambda-3) r_{s}+4 \lambda r\right)}{4 \lambda r}+\frac{i \lambda}{2 r \omega^{2}}, \quad \mathcal{T}(r) \equiv \frac{(1-2 \lambda) r_{s}+2(1+2 \lambda) r}{4 r \omega}, \\
& \mathcal{U}(r) \equiv r^{2}+\frac{2 \lambda-3}{4 \lambda} r_{s} r, \quad \mathcal{V}(r) \equiv \frac{i r}{2 \omega}
\end{aligned}
$$

This leads to the new system

$$
\frac{\mathrm{d} \tilde{Y}}{\mathrm{~d} r}=\tilde{M}(r) \tilde{Y}, \quad \tilde{M}(r)=\left(\begin{array}{cc}
i \omega & 0 \\
0 & -i \omega
\end{array}\right)+\left(\begin{array}{cc}
-1+i \omega r_{s} & 0 \\
0 & -1-i \omega r_{s}
\end{array}\right) \frac{1}{r}+\mathcal{O}\left(\frac{1}{r^{2}}\right),
$$

which is diagonal and whose solution is

$$
\tilde{Y}(r)=\left(\begin{array}{l}
c_{-} e^{-i \omega r} r^{-1-i \omega r_{s}} \\
c_{+} e^{+i \omega r} r^{-1+i \omega r_{s}}
\end{array}\right)(1+\mathcal{O}(1 / r))=\frac{1}{r}\left(\begin{array}{c}
c_{-} e^{-i \omega r_{*}} \\
c_{+} e^{+i \omega r_{*}}
\end{array}\right)(1+\mathcal{O}(1 / r)) .
$$

This result is very similar to that obtained for axial perturbations (3.18), even though the asymptotic expansion of the matrix $M$ is rather different. In terms of the original functions, we find

$$
K(r)=\frac{i}{\omega} H_{1}(r)=i\left(c_{-} e^{-i \omega r_{*}}+c_{+} e^{+i \omega r_{*}}\right)(1+\mathcal{O}(1 / r)),
$$

which agree with 2.30 (with $c_{-}=-\omega \mathcal{B}_{\infty}$ and $c_{+}=\omega \mathcal{A}_{\infty}$ ). 


\section{Asymptotic analysis at the black hole horizon}

We finally turn to the near-horizon behaviour of polar modes. The expansion of the matrix (3.35) in terms of the small parameter $\varepsilon \equiv r-r_{s}$ yields

$$
M(\varepsilon)=\frac{1}{\varepsilon^{2}} M_{2}+\frac{1}{\varepsilon} M_{1}+M_{0}+\mathcal{O}(\varepsilon), \quad M_{2}=\left(\begin{array}{cc}
0 & 0 \\
\gamma_{2} & 0
\end{array}\right), M_{1}=\left(\begin{array}{cc}
\alpha_{1} & 0 \\
\gamma_{1} & \delta_{1}
\end{array}\right), M_{0}=\left(\begin{array}{cc}
\alpha_{0} & \beta_{0} \\
\gamma_{0} & \delta_{0}
\end{array}\right),(3
$$

where only a few of the coefficients $\alpha_{I}, \beta_{I}$ and $\gamma_{I}$ will be needed explicitly.

Once more, the dominant $M_{2}$ is a nilpotent matrix and, as in the axial case, we use the transformation

$$
Y=P_{(1)} Y^{(1)} \quad \text { with } \quad P_{(1)}(\varepsilon) \equiv\left(\begin{array}{cc}
1 & 0 \\
0 & 1 / \varepsilon
\end{array}\right)
$$

which gives the new system

$$
\frac{\mathrm{d} Y^{(1)}}{\mathrm{d} \varepsilon}=M^{(1)} Y^{(1)}, \quad M^{(1)}(\varepsilon)=\frac{1}{\varepsilon}\left(\begin{array}{cc}
\alpha_{1} & \beta_{0} \\
\gamma_{2} & 1+\delta_{1}
\end{array}\right)+\mathcal{O}(1),
$$

with the coefficients

$$
\alpha_{1}=-\left(1+\delta_{1}\right)=\frac{1+\lambda-2 r_{s}^{2} \omega^{2}}{3+2 \lambda}, \quad \beta_{0}=\frac{2 i}{r_{s}^{2}} \frac{(\lambda+1)^{2}+r_{s}^{2} \omega^{2}}{3+2 \lambda}, \quad \gamma_{2}=\frac{i r_{s}^{2}}{2} \frac{1+4 r_{s}^{2} \omega^{2}}{3+2 \lambda} .
$$

The leading matrix can now be diagonalised via the transformation

$$
Y^{(1)}=P_{(2)} Y^{(2)}, \quad \text { with } \quad P_{(2)}=\left(\begin{array}{cc}
\alpha-\beta & \alpha+\beta \\
1 & 1
\end{array}\right) \quad \text { and } \quad \alpha=\frac{\alpha_{1}}{\gamma_{2}}, \quad \beta=\frac{i \omega r_{s}}{\gamma_{2}} .
$$

leading to the system

$$
\frac{\mathrm{d} Y^{(2)}}{\mathrm{d} \varepsilon}=M^{(2)} Y^{(2)}, \quad M^{(2)}=\frac{1}{\varepsilon}\left(\begin{array}{cc}
-i \omega r_{s} & 0 \\
0 & i \omega r_{s}
\end{array}\right)+\mathcal{O}(1)
$$

Note that this expression is extremely simple and does not involve $\lambda$, as expected, even though it appears explicitly in $M^{(1)}$. We obtain immediately the asymptotic behaviour of $X^{(2)}$ near the horizon

$$
Y^{(2)}(\varepsilon)=(1+\mathcal{O}(\varepsilon))\left(\begin{array}{l}
c_{-} e^{-i \omega r_{*}} \\
c_{+} e^{+i \omega r_{*}}
\end{array}\right)
$$

which reproduces the same result as for the axial mode (3.31). In terms of the original gravitational functions $H_{1}(r)$ and $K(r)$, using the transformation $Y=P_{(1)} P_{(2)} Y^{(2)}$, we recover the result 2.32$)$, with

$$
c_{+}=\frac{i}{2} r_{s}\left(1-2 i \omega r_{s}\right) \mathcal{A}_{\mathrm{hor}} \quad c_{-}=-\frac{i}{2} r_{s}\left(1+2 i \omega r_{s}\right) \mathcal{B}_{\mathrm{hor}}
$$

This completes our study of all asymptotic behaviours of Schwarzschild perturbations, demonstrating that one can recover the standard results directly from the linearised Einstein's equations, without resorting to the Schrödinger-like reformulation of the system. 


\section{Quasi-Normal modes}

Several powerful numerical methods have been developed for the computation of quasinormal modes when the system is of the form (1.1), but these methods cannot be directly applied to the more general first-order system we are dealing with. In this section, we use a simple numerical method to show how the Schwarzschild quasinormal modes can be recovered numerically, using directly the first-order system instead of the Schrödinger equation (2.18). We restrict ourselves to the polar modes and consider the system 3.34 3.35). The computation of the axial quasinormal modes would be completely similar.

By definition of the quasi-normal modes, we impose that the solutions are outgoing at spatial infinity and ingoing at the horizon, which means, using the results of section IIIB, that the two components of the vector $Y$ satisfy

$$
\begin{aligned}
Y_{1}(r) \equiv K(r) & =K_{\infty}(r) e^{i \omega r_{*}}=\tilde{K}_{\infty}(r) e^{i \omega r} r^{i \omega r_{s}} \\
& =K_{\mathrm{h}}(r) e^{-i \omega r_{*}}=\tilde{K}_{\mathrm{h}}(r)\left(r-r_{s}\right)^{-i \omega r_{s}}
\end{aligned}
$$

where $K_{\infty}\left(\right.$ and $\left.\tilde{K}_{\infty}\right)$ is finite at infinity while $K_{\mathrm{h}}\left(\right.$ and $\left.\tilde{K}_{\mathrm{h}}\right)$ is finite at the horizon, and also

$$
\begin{aligned}
Y_{2}(r) \equiv H_{1}(r) / \omega & =H_{\infty}(r) r e^{i \omega r_{*}}=\tilde{H}_{\infty}(r) e^{i \omega r} r^{1+i \omega r_{s}} \\
& =H_{\mathrm{h}}(r) \varepsilon^{-1} e^{-i \omega r_{*}}=\tilde{H}_{\mathrm{h}}(r)\left(r-r_{s}\right)^{-1-i \omega r_{s}},
\end{aligned}
$$

where again $H_{\infty}\left(\right.$ and $\left.\tilde{H}_{\infty}\right)$ is finite at infinity while $H_{\mathrm{h}}\left(\right.$ and $\left.\tilde{H}_{\mathrm{h}}\right)$ is finite at the horizon.

Therefore, we look for solutions of 3.343 .35 using the ansatz

$$
K(r)=e^{i \omega r} r^{i \omega r_{s}}\left(\frac{r-r_{s}}{r}\right)^{-i \omega r_{s}} f_{K}(r), \quad H_{1}(r)=e^{i \omega r} r^{1+i \omega r_{s}}\left(\frac{r-r_{s}}{r}\right)^{-1-i \omega r_{s}} f_{H}(r),
$$

where the functions $f_{K}$ and $f_{H}$ are supposed to be finite (hence bounded) both at the horizon and at spatial infinity, in agreement with the required boundary conditions. Furthermore, we introduce the new variable

$$
u=\frac{2 r_{s}}{r}-1,
$$

so that the black hole horizon is located at $u=1$ and spatial infinity at $u=-1$. Each function entering in the equations (3.54) is now treated as a function of $u$ and the system of equations 3.343 .35 can be expressed in the form

$$
\begin{aligned}
& \mathcal{P}_{11}(u) f_{K}(u)+\mathcal{P}_{12}(u) f_{H}(u)+\mathcal{Q}_{1}(u) f_{K}^{\prime}(u)=0, \\
& \mathcal{P}_{21}(u) f_{K}(u)+\mathcal{P}_{22}(u) f_{H}(u)+\mathcal{Q}_{2}(u) f_{H}^{\prime}(u)=0,
\end{aligned}
$$

where a prime denotes here a derivative with respect to $u$, and the functions $\mathcal{P}_{i j}$ and $\mathcal{Q}_{i}$ are polynomials in $u$. This is possible because the matrix $M$ given in (3.35) contains only rational fractions of $r$.

In order to solve the system (3.56) numerically, we adapt the spectral method presented in [22] and we decompose $f_{K}(u)$ and $f_{H}(u)$ onto a basis of Chebyshev polynomials. The facts that the functions $\mathcal{P}_{i j}$ and the $\mathcal{Q}_{i}$ are polynomials (hence $C^{\infty}$-functions) and the Chebyshev polynomials are bounded at the boundaries ensure the boundedness of $f_{K}(u)$ and $f_{H}(u)$ which is sufficient to enforce the required boundary conditions. This is called a "behavioural" boundary condition [23].

Then, any smooth and continuous complex-valued function $g(u)$ defined on the interval $[-1,1]$ can be written as an infinite sum of Chebyshev polynomials $T_{n}(u)$,

$$
g(u)=\sum_{n=0}^{\infty} g_{n} T_{n}(u)
$$


where $g_{n}$ are complex coefficients. We can approximate the function $g$ by truncating this series at a given order $N$, the approximation getting better as $N$ is increased. Hence, we decompose the two functions $f_{K}$ and $f_{H}$ as follows,

$$
f_{K}(u) \approx \sum_{n=0}^{N} \alpha_{n} T_{n}(u), \quad f_{H}(u) \approx \sum_{n=0}^{N} \beta_{n} T_{n}(u)
$$

where $\alpha_{n}$ and $\beta_{n}$ are complex coefficients. Notice that the symbol $\approx$ means that we truncated the series at an order $N$, then the equality is not exact.

The next step is to express the differential system (3.56) as a linear system for the coefficients $\alpha_{n}$ and $\beta_{n}$, which is always possible due to fundamental relations satisfied by Chebyshev polynomials ${ }^{5}$. As a consequence, the differential system (3.56) can be recast as the following system of algebraic equations

$$
M_{N}(\omega) V_{N}\left(\alpha_{n}, \beta_{n}\right)=0,
$$

where $M_{N}$ is a $2(N+1) \times 2(N+1)$ matrix whose expansion in powers of $\omega$ reads

$$
M_{N}(\omega)=M_{N[0]}+M_{N[1]} \omega+M_{N[2]} \omega^{2}
$$

while the $2(N+1)$-dimensional vector $V_{N}\left(\alpha_{n}, \beta_{n}\right)$ is such that

$$
{ }^{T} V_{N}\left(\alpha_{n}, \beta_{n}\right) \equiv\left(\alpha_{0}, \cdots, \alpha_{N}, \beta_{0}, \cdots, \beta_{N}\right)
$$

Following [22], we can reformulate this system as

$$
\tilde{M}_{N}(\omega) \tilde{V}_{N}\left(\alpha_{n}, \beta_{n}\right)=0
$$

where the matrix $\tilde{M}_{N}$ is now of dimension $4(N+1)$ and defined by

$$
\tilde{M}_{N}=\tilde{M}_{N[0]}+\tilde{M}_{N[1]} \omega \quad \text { and } \quad \tilde{M}_{N[0]}=\left(\begin{array}{cc}
M_{N[0]} & M_{N[1]} \\
0 & I
\end{array}\right), \quad \tilde{M}_{N[1]}=\left(\begin{array}{cc}
0 & M_{N[2]} \\
-I & 0
\end{array}\right) .
$$

Finding the values of $\omega$ such that the system (3.63) is nontrivial is called a generalised eigenvalues problem and can be done by a numerical engine such as Mathematica or Scipy. In practice, we have computed the eigenvalues for different values of $N$ and identified the ones that (almost) coincide when $N$ varies. There are also nonphysical spurious modes (due to the finite size approximation), which strongly depend on $N$ and must be discarded. The quasinormal modes thus identified, plotted in Fig.(2), coincide with the well-known first quasi-normal modes of Schwarzschild.

This result demonstrates that it is feasible to compute quasinormal modes directly from the first-order system, even if our numerical approach is rather crude and gives a very low precision with respect to the sophisticated methods used in the traditional approach.

\footnotetext{
${ }^{5}$ The Chebyshev polynomials satisfy the properties

$$
T_{n}^{\prime}(u)=\sum_{m-n=2 k+1} \frac{2 m}{1+\delta_{n 0}} T_{m}(u), \quad\left(u T_{n}\right)(u)=\sum_{m} \frac{1}{2}\left(\left(1+\delta_{n, 1}\right) \delta_{n-1, m}+\delta_{n+1, m}\right) T_{m}(u),
$$
}

where $\delta_{m, n}$ is the Kronecker symbol and $k \in \mathbb{N}$ in the first sum. 


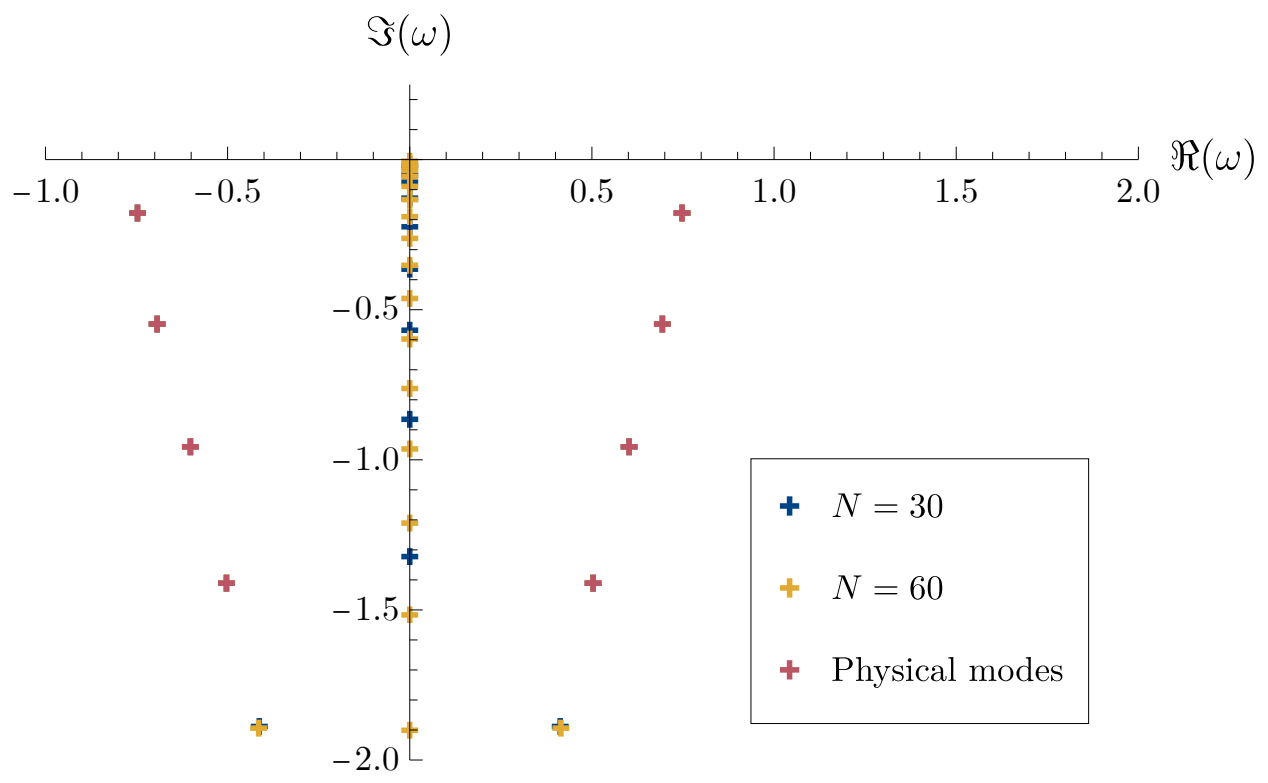

FIG. 2 Quasinormal modes numerically found by Mathematica for $r_{s}=1$ and $\ell=2(\lambda=2)$. The blue dots are generalised eigenvalues for $N=30$, the orange dots generalised eigenvalues for $N=60$, and the red dots are the modes detected (eigenvalues that change by a factor $10^{-3}$ or less). All the dots present on the imaginary axis correspond to spurious modes. We observe a symmetry with respect to the imaginary axis. The positions of the first modes are $\omega_{0}= \pm 0.747-0.178 i, \omega_{1}= \pm 0.693-0.548 i$ and $\omega_{2}= \pm 0.602-0.957 i$.

\section{GENERAL ANALYSIS}

As we have seen in the previous section, it is possible to compute the quasi-normal modes of black holes in general relativity without reformulating the linearised Einstein equations in terms of a Schrödinger-like equation. The advantage of this method is that it can be straightforwardly generalised to the study of black holes in theories of modified gravity where it might be difficult or impossible to reduce the linearised equations to a Schrödinger-like form.

In this section, we present a systematic algorithm for a generic first-order system of the form (3.7), which has been developed in the mathematics literature, first in [18] and more recently in [19 21, 24, 25]. The various steps of the algorithm presented in this section are summarised in the flowchart diagram depicted in Appendix C.

\section{A. Asymptotic solution: overview}

We consider a general system of first-order ordinary differential equations of the form

$$
\frac{\mathrm{d} Y}{\mathrm{~d} z}=M(z) Y
$$

where $Y$ is a $n$-dimensional column vector, $M$ an $n \times n$-dimensional matrix and $z$ a real variable defined in some interval. In the following, we will consider only the asymptotic behaviour when $z \rightarrow+\infty$, but it is straightforward to extend the algorithm near a finite value $z_{0}$ where the system is singular, by a suitable change of the variable $z$.

We then assume that one can expand $M$ in powers of $z$, up to some order (depending on the 
required precision of the asymptotic expansion) as follows,

$$
M(z)=M_{r} z^{r}+\cdots+M_{0}+\ldots M_{r-f} z^{r-f}+\mathcal{O}\left(z^{r-f-1}\right)=z^{r} \sum_{k=0}^{f} M_{r-k} z^{-k}+\mathcal{O}\left(z^{r-f-1}\right),
$$

where the integer $r$ is called the Poincaré rank of the system, and the $M_{i}$ are $z$-independent matrices. In most $\operatorname{cases}^{6}$, the general solution to the system (4.1) admits an asymptotic expansion of the form [18]

$$
Y(z)=e^{\boldsymbol{\Upsilon}(z)} r^{\boldsymbol{\Delta}} \mathbf{F}(z) Y_{0}
$$

where $Y_{0}$ is a constant vector, corresponding to $n$ integration constants, $\boldsymbol{\Upsilon}$ is a diagonal matrix whose coefficients are polynomials of degree at most $r+1, \boldsymbol{\Delta}$ is a constant diagonal matrix and $\mathbf{F}(z)$ is a matrix which is regular at infinity.

The goal of the algorithm presented below is to determine explicitly the expression (4.3) up to some irrelevant sub-leading terms. As we have already seen in the previous section, the guiding principle in order to obtain this expression is to fully diagonalise the differential system, up to the appropriate order, by using iteratively transformations of the vector $Y$ into a new vector $\tilde{Y}$, of the form

$$
Y(z)=P(z) \tilde{Y}(z)
$$

where $P$ is an invertible matrix. The system (4.1) is then transformed into a new but equivalent differential system, given by

$$
\frac{\mathrm{d} \tilde{Y}}{\mathrm{~d} z}=\tilde{M}(z) \tilde{Y}, \quad \tilde{M}(z) \equiv P^{-1} M P-P^{-1} \frac{\mathrm{d} P}{\mathrm{~d} z} .
$$

The end point of this procedure is a system where the matrix coefficients in the expansion of the form $(4.2)$ are diagonal at each order. It is then immediate to integrate the system and to find the solution in the form (4.3), as discussed in section III A.

In the following subsections, we describe the algorithm step by step. We have also inserted two subsections that contain examples chosen to illustrate some of the finer points of the algorithm. The algorithm contains several branches, depending on whether the leading term $M_{r}$ in the expansion of $M(z)$ is diagonalisable or not.

\section{B. Case 1: the leading term is diagonalisable}

The simplest situation is when the leading matrix $M_{r}$ is diagonalisable, with each eigenvalue of multiplicity 1. In this case, one first uses the transformation $Y=P_{(1)} Y^{(1)}$ where $P_{(1)}$ is a constant matrix that diagonalises $M_{r}$, which gives the new system

$$
\frac{\mathrm{d} Y^{(1)}}{\mathrm{d} z}=M^{(1)} Y^{(1)}, \quad M^{(1)}(z)=D_{r} z^{r}+M_{r-1}^{(1)} z^{r-1}+\cdots+M_{0}^{(1)}+M_{-1}^{(1)} \frac{1}{z}+\mathcal{O}\left(\frac{1}{z^{2}}\right),
$$

where the matrix $D_{r}$ is diagonal.

\footnotetext{
${ }^{6}$ Note that, in some cases, the variable $z$ in the expression 4.3 differs from the variable $z$ in the original system (4.1), because a change of variable is necessary, as will be discussed around Eq. (4.21). Morever, the special case where $M(z)=M_{-1} / z+\mathcal{O}\left(z^{-2}\right)$ with $M_{-1}$ nilpotent leads to a ln $z$ behaviour at large $z$, as discussed at the end of section IV C
} 
One then seeks to transform the next-to-leading matrix $M_{r-1}^{(1)}$ into a diagonal matrix (if it is not already) without affecting the diagonal form of the leading order. This can be accomplished with a new transformation

$$
Y^{(1)}=P_{(2)} Y^{(2)}, \quad P_{(2)}(z)=I+\frac{1}{z} \Xi_{(2)},
$$

where $\Xi_{(2)}$ is a constant matrix. Indeed, this yields the new system

$$
\frac{\mathrm{d} Y^{(2)}}{\mathrm{d} z}=M^{(2)} Y^{(1)}, \quad M^{(2)}(z)=D_{r} z^{r}+D_{r-1} z^{r-1}+M_{r-2}^{(2)} z^{r-2}+\cdots+M_{-1}^{(2)} \frac{1}{z}+\mathcal{O}\left(\frac{1}{z^{2}}\right),
$$

with

$$
D_{r-1}=M_{r-1}^{(1)}+\left[D_{r}, \Xi^{(2)}\right]
$$

which is imposed to be diagonal via an appropriate choice ${ }^{7}$ for $\Xi_{(2)}$. Furthermore, $D_{r-1}$ is the diagonal part of $M_{r-1}^{(1)}$.

One can proceed similarly to "diagonalise" all the other terms, order by order, until one gets a system of the form ${ }^{8}$

$$
\frac{\mathrm{d} Y^{(r+2)}}{\mathrm{d} z}=M^{(r+2)} Y^{(r+2)}, \quad M^{(r+2)}(z)=D_{r} z^{r}+\cdots+D_{0}+D_{-1} \frac{1}{z}+\mathcal{O}\left(\frac{1}{z^{2}}\right),
$$

where all matrices are diagonal up to order $1 / z$. The system can then be immediately integrated, to yield

$$
Y^{(r+2)}(z)=e^{\Upsilon(z)} z^{\Delta} \mathbf{F}(z) Y_{0}, \quad \boldsymbol{\Delta} \equiv D_{-1}, \quad \mathbf{\Upsilon}(z) \equiv D_{r} \frac{z^{r+1}}{r+1}+\cdots+D_{0} z
$$

where $Y_{0}$ is a constant vector.

The asymptotic expansion of the original vector $Y$ can be simply deduced from the combined transformations, i.e.

$$
Y=P_{(1)} P_{(2)} \cdots P_{(r+2)} Y^{(r+2)} .
$$

Since the $P_{(j)}$ are polynomials of $1 / z, Y$ has exactly the same exponential behaviour (in its asymptotic expansion) as $Y^{(r+2)}$.

The above procedure is not directly applicable if the leading matrix $M_{r}$ has eigenvalues of multiplicity higher than one. In such a case, writing $M_{r}$ in a block diagonal form, with eigenvalues $\lambda_{i}$ of multiplicity $m_{i}$, one applies a transformation

$$
Y^{(1)}=P_{(2)} Y^{(2)}
$$

\footnotetext{
${ }^{7}$ To find $\Xi$ such that the matrix $\tilde{D}=M+[D, \Xi]$ is diagonal, $M$ being arbitrary and $D$ diagonal, one notices that $[D, \Xi]_{i j}=\left(d_{i}-d_{j}\right) \Xi_{i j}$ where $d_{i}$ are the eigenvalues of $D$. Consequently, $\tilde{D}$ is given by the diagonal component of $M$ and the coefficients of $\Xi$ satisfy $\left(d_{i}-d_{j}\right) \Xi_{i j}+M_{i j}=0$, which always admit at least one solution for each $\Xi_{i j}$ as long as all $d_{i}$ are different.

${ }^{8}$ Note that we could have proceeded in a single step by introducing the new variable $\tilde{Y}$ defined by $Y=P(z) \tilde{Y}$ with $P(z)=P_{0}+\frac{1}{z} P_{1}+\cdots+\frac{1}{z^{r+1}} P_{r+1}$ and determining the constant matrices $P_{j}$ so that $\tilde{M}(z)$ is equal to 4.9 . The calculation we have just done proves this is possible with $\tilde{Y}=Y^{(r+2)}$.
} 
where $P_{(2)}$ has the same block structure as $M_{r}$, with the blocks $B_{i}$ of size $m_{i} \times m_{i}$ defined as $B_{i}=\exp \left(\frac{\lambda_{i}}{r+1} z^{r+1}\right)$ if $m_{i} \geq 2$ and $B_{i}=1$ if $m_{i}=1$. For example, if the leading matrix is $M_{r}=$ $\operatorname{Diag}\left(\lambda_{1}, \lambda_{1}, \lambda_{2}\right)$, with $r=1$, then the transformation is $P_{(2)}=\operatorname{Diag}\left(\exp \left(\lambda_{1} \frac{z^{2}}{2}\right), \exp \left(\lambda_{1} \frac{z^{2}}{2}\right), 1\right)$.

Such a transformation puts the multi-dimensional blocks to zero, allowing one to pursue the algorithm with the subleading terms. One must however be careful when coming back to the original variable $Y^{(1)}$, since the transformation $P_{(2)}$ will greatly affect the computed asymptotic behaviour.

\section{Case 2: the leading term is non-diagonalisable, similar to a single-block Jordan matrix}

Solving asymptotically a system where the dominant term $M_{r}$ is not diagonalisable is more challenging. The basic idea consists in finding a transformation where the leading term of the new matrix becomes diagonalisable. This can be done by reducing progressively the Poincaré rank of the system until the leading term is diagonalisable, in which case the procedure of the previous subsection becomes applicable. If the leading term never gets diagonalisable down to the rank $r=-1$, then the general formula (4.3) for the asymptotic expansion is not valid but the system can nevertheless be integrated explicitly.

The reduction of the Poincaré rank together with the diagonalisation of the leading term is done in different steps, which we now describe, first when the leading term is similar to a Jordan matrix with a single block. The case of a Jordan matrix with several blocks will be discussed later, in section IVE

\section{Step 1. Transformation to a Jordan block}

Starting from the asymptotic expansion (4.2) of the matrix $M$, we use the transformation $X=P_{(1)} X^{(1)}$ to write $M_{r}^{(1)}=P_{(1)}^{-1} M_{r} P_{(1)}$ in a Jordan canonical form (although with a lower triangular matrix). We assume here that $M_{r}^{(1)}$ contains a single (lower triangular) Jordan block with eigenvalue $\lambda$, i.e. of the form

$$
M_{r}^{(1)}=\left(\begin{array}{ccccc}
\lambda & 0 & \cdots & \\
1 & \lambda & 0 & \ldots & \\
0 & 1 & \lambda & 0 & \ldots \\
\vdots & & & &
\end{array}\right) \equiv \lambda I+J(n),
$$

where $J(n)$ has the property to be nilpotent (we recall that $n$ is the dimension of the matrix).

\section{Step 2. Transformation to a nilpotent matrix}

We then apply the transformation

$$
Y^{(1)}=P_{(2)} Y^{(2)}, \quad P_{(2)}(z) \equiv \exp \left(\frac{\lambda}{r+1} z^{r+1}\right) I,
$$


which renders the leading term nilpotent ${ }^{9}$

$$
M^{(2)}(z)=J(n) z^{r}+M_{r-1}^{(2)} z^{r-1}+\cdots+M_{0}^{(2)}+M_{-1}^{(2)} \frac{1}{z}+\mathcal{O}\left(\frac{1}{z^{2}}\right) .
$$

Step 3. Normalisation and reduction of the Poincaré rank

The next step consists in reducing the Poincaré rank of the system by using the transition matrix

$$
P(z)=D(n, z) \equiv\left(\begin{array}{cccccc}
1 & 0 & 0 & \cdots & \cdots & 0 \\
0 & z & 0 & \cdots & \cdots & 0 \\
0 & 0 & z^{2} & 0 & \cdots & 0 \\
\vdots & & & \ddots & & \vdots \\
0 & \cdots & & & \cdots & z^{n-1}
\end{array}\right)
$$

which satisfies the useful property

$$
P^{-1} J(n) P=\frac{1}{z} J(n) .
$$

A transformation with the above $P$ will thus reduce the order of the leading term $J(n) z^{r}$, but will also affect the sub-dominant terms in the expansion 4.16) of $M^{(2)}$, in particular $M_{r-1}^{(2)}$ which could generate terms whose order is higher than $r-1$ in the new matrix.

To avoid this situation, we need first to "normalise" the system, with the transformation

$$
P_{(3)}(z)=I+\frac{1}{z} \Lambda_{(3)},
$$

where $\Lambda_{(3)}$ is a constant matrix, chosen such that such that the next-to-leading order matrix $M_{r-1}^{(3)}$ in the new matrix expansion contains only zeros except possibly in the first row. Let us stress that this transformation leaves the leading term of the expansion unchanged. The new system associated with $M^{(3)}$ is said to be normalised.

One can then perform the transformation generated by the transition matrix

$$
P_{(4)}(z)=D(n, z),
$$

which, in most cases, gives a reduced Poincaré rank. There are however a few exceptions where the reduction does not work. These special cases require a more general transformation, with a transition matrix of the form

$$
P_{(4)}(z)=D\left(n, z^{p / q}\right), \quad(1 \leq p \leq q \leq n)
$$

where $p$ and $q$ are co-prime integers. For example, when $n=4$, the possible choices are $\{1 / 4,1 / 3,1 / 2,2 / 3,3 / 4,1\}$, where the last value corresponds to the generic case 4.20). To identify the appropriate value of $p / q$, one must test successively the possible values, in decreasing order,

9 This follows from the relation

$$
P_{(2)}^{-1}\left(z^{r}(\lambda I+J(n)) P_{(2)}-P_{(2)}^{-1} \frac{\mathrm{d} P_{(2)}}{\mathrm{d} z}=z^{r}\left(M_{r}^{(1)}-\lambda I\right)=z^{r} J(n) .\right.
$$


until the transformation (4.21) effectively leads to a system with a lower Poincaré rank. The corresponding value of $p / q$ is said to be "admissible". In practice, this can be understood as a change of variable ${ }^{10}, z$ being replaced by $u=z^{p / q}$.

\section{Step 4. Diagonalisable or not diagonalisable?}

The next step depends on the nature of the system $\left(Y^{(4)}, M^{(4)}\right)$, which possesses a lower Poincaré rank than the initial system. If the leading term of $M^{(4)}$ is diagonalisable, one proceeds as in section IVB.

If $M^{(4)}$ is not diagonalisable, one needs to reduce again the Poincaré rank of the system, unless one has already reached $r=-1$, in which case one can jump directly to the next paragraph. Otherwise, one must distinguish the following different cases.

- If the leading term is similar to a single-block Jordan matrix and we took $p / q=1$ in the previous step, we repeat the procedure of this subsection.

- If the leading term is similar to a single-block Jordan matrix but we took $p / q<1$ in the previous step, we discard the last step, and start again with the normalised system $M^{(3)}$. However, this time, we normalise the system up to second order: after having normalised $M_{-1}$, we repeat the procedure with $z^{2}$ instead of $z$ in $P_{(3)} 4.19$ and require that $M_{-2}$ has a specific form. Details can be found in [19]. If necessary, one can pursue the normalisation to higher orders.

- If the Jordan canonical form of the leading term contains several blocks, we go to section IVE

Eventually we obtain either a system with a diagonalisable leading term, which can be solved following section IV B, or a system of Poincaré rank $r=-1$ with a nilpotent leading term. In the latter case, the solution is equivalent to a polynomial of $\ln z$ at large $z$. Indeed, a system of the form

$$
\frac{\mathrm{d} Y}{\mathrm{~d} z}=\frac{\mu_{0}}{z}\left(\begin{array}{ccccc}
0 & 0 & \cdots & & \\
1 & 0 & 0 & \cdots & \\
0 & 1 & 0 & 0 & \cdots \\
\vdots & & & &
\end{array}\right) Y
$$

where $\mu_{0}$ is an arbitrary constant, is easily integrated. The components $Y_{i}$ (for $1 \leq i \leq n$ ) are obtained iteratively and are given by $Y_{1}(z)=\xi_{1}, Y_{2}(z)=\xi_{1} \ln z+\xi_{2}$ and more generally,

$$
Y_{i}(z)=\sum_{j=1}^{i} \frac{\xi_{j}}{(i-j) !}\left(\mu_{0} \ln z\right)^{i-j},
$$

where the $\xi_{i}$ are $n$ constants of integration. All the components of $Y$ are thus polynomials of $\ln z$ at large $z$.

${ }^{10}$ In this case, the asymptotic expansion of the solution may have an exponential behaviour where the argument $Q(z)$ is not a polynomial of $z$ but rather a polynomial of $z^{1 / q}$. 


\section{An example with a nilpotent leading term}

Let us give a concrete example of the procedure used for systems with a nilpotent leading term. We consider the two-dimensional system defined by

$$
\frac{\mathrm{d} Y}{\mathrm{~d} z}=M(z) Y, \quad M(z)=\left(\begin{array}{cc}
0 & 1 \\
0 & 0
\end{array}\right) z^{2}+\left(\begin{array}{cc}
1 & 0 \\
0 & -1
\end{array}\right)
$$

and let us determine its asymptotic solution at large $z$, following the algorithm described above.

We first put the leading term in its lower triangular Jordan form:

$$
P_{(1)}=\left(\begin{array}{ll}
0 & 1 \\
1 & 0
\end{array}\right) \quad \Longrightarrow \quad M^{(1)}(z)=\left(\begin{array}{ll}
0 & 0 \\
1 & 0
\end{array}\right) z^{2}+\left(\begin{array}{cc}
-1 & 0 \\
0 & 1
\end{array}\right) \text {. }
$$

Since the leading term is already nilpotent, step 2 is irrelevant. Moreover, the system is already normalised since the next-to-leading order term vanishes.

We can thus move directly to the reduction of the order of the system and consider the transformation of the form 4.17):

$$
P_{(2)}(z)=\left(\begin{array}{ll}
1 & 0 \\
0 & z
\end{array}\right) \quad \Longrightarrow \quad M^{(2)}(z)=\left(\begin{array}{ll}
0 & 0 \\
1 & 0
\end{array}\right) z+\left(\begin{array}{cc}
-1 & 0 \\
0 & 1
\end{array}\right)
$$

The order has been reduced but the leading term is still nilpotent. Since the reduction was obtained via a transformation with $p / q=1$, we continue the process by doing a new iteration of the algorithm. We first normalise the system with a transformation of the form 4.19,

$$
P_{(3)}(z)=I+\frac{1}{z}\left(\begin{array}{cc}
0 & -1 \\
0 & 0
\end{array}\right) \quad \Longrightarrow \quad M^{(3)}(z)=\left(\begin{array}{ll}
0 & 0 \\
1 & 0
\end{array}\right) z+\left(\begin{array}{cc}
0 & 1 \\
0 & -1
\end{array}\right) \frac{1}{z}+\left(\begin{array}{cc}
0 & -2 \\
0 & 0
\end{array}\right) \frac{1}{z^{2}},
$$

and again reduce the order of the system with the transformation

$$
P_{(4)}(z)=\left(\begin{array}{ll}
1 & 0 \\
0 & z
\end{array}\right) \quad \Longrightarrow \quad M^{(4)}(z)=\left(\begin{array}{ll}
0 & 1 \\
1 & 0
\end{array}\right)+\left(\begin{array}{ll}
0 & -2 \\
0 & -2
\end{array}\right) \frac{1}{z} \text {. }
$$

The leading term is now diagonalisable. We diagonalise it explicitly, via

$$
P_{(5)}=\left(\begin{array}{cc}
-1 & 1 \\
1 & 1
\end{array}\right) \quad \Longrightarrow \quad M^{(5)}(z)=\left(\begin{array}{cc}
-1 & 0 \\
0 & 1
\end{array}\right)+\left(\begin{array}{cc}
0 & 0 \\
-2 & -2
\end{array}\right) \frac{1}{z}
$$

then we diagonalise the next-to-leading term, with a transformation of the form 4.6,

$$
P_{(6)}(z)=\left(\begin{array}{cc}
1 & 0 \\
1 / z & 1
\end{array}\right) \quad \Longrightarrow \quad M^{(6)}(z)=\left(\begin{array}{cc}
-1 & 0 \\
0 & 1
\end{array}\right)+\left(\begin{array}{cc}
0 & 0 \\
0 & -2
\end{array}\right) \frac{1}{z}+\mathcal{O}\left(\frac{1}{z^{2}}\right)
$$

We have thus managed to fully diagonalise the system, which immediately gives us the asymptotic solution

$$
Y^{(6)}(z)=(1+\mathcal{O}(1 / z))\left(\begin{array}{cc}
\exp (-z) & 0 \\
0 & \frac{1}{z^{2}} \exp (z)
\end{array}\right) Y_{0}, \quad Y_{0} \equiv\left(\begin{array}{l}
\xi_{1} \\
\xi_{2}
\end{array}\right),
$$

where $Y_{0}$ is a constant column vector. As a consequence, to obtain the behaviour of $Y$ in the original system, we use the combined transformations

$$
Y=\left(\prod_{j=1}^{6} P_{(j)}\right) Y^{(6)}
$$


which implies

$$
Y(z)=(1+\mathcal{O}(1 / z))\left(\begin{array}{c}
\xi_{1} \exp (-z) z^{2}+\xi_{2} \exp (z) \\
-2 \xi_{1} \exp (-z)
\end{array}\right)
$$

For this particular example, it turns out that the original system (4.24) can be solved exactly, with the solution

$$
Y(z)=\left(\begin{array}{c}
\frac{1}{2} \xi_{1} \exp (-z)\left(1+2 z+2 z^{2}\right)+\xi_{2} \exp (z) \\
-2 \xi_{1} \exp (-z)
\end{array}\right) .
$$

One can thus check that the asymptotic solution (4.33) agrees with the asymptotic behaviour of the exact solution.

\section{E. Case 3: $M_{r}$ is similar to a Jordan matrix with several blocks}

We now briefly discuss (without entering into too many details, which can be found in [19]) the more general case where $M_{r}$ is block diagonalisable and its canonical Jordan form admits several Jordan blocks. The first two steps of section IVC still apply to this case and one can find a transformation (with a constant matrix $P$ ) such that the new system associated with $M^{(2)}$ (we use the same notation as in section IV C has a block diagonal leading term $M_{r}^{(2)}$ with Jordan lower triangular blocks, each block being either nilpotent or 1-dimensional:



The Jordan form is chosen so that the blocks $J(n)$ are ordered by decreasing size $\left(n_{1} \geq n_{2} \geq \cdots\right)$. We will use this block structure as a layout for the block structure of the other matrices that appear in the expansion of $M^{(2)}$. And each block will be denoted by two indices, $(K L)$, corresponding to a submatrix of dimensions $n_{K} \times n_{L}$.

The principle of the diagonalisation procedure is similar to what was done in sections $\mathrm{IVB}$ and IVC. However, it is now possible to have both diagonalisable blocks and nilpotent blocks. Those must be dealt with separately to get the full asymptotic behaviour of the system. In order to do this, one can generalise the order-by-order procedure of section IVB: this is called the "Splitting Lemma" in [19]. It is not detailed here, but can be understood by considering blocks instead of scalars in the computations of section IV B ${ }^{11}$.

${ }^{11}$ In the case where $M_{r}^{(2)}$ consists of a 2-block Jordan matrix, one would use a transformation of the form

$$
P=\left(\begin{array}{cc}
I & \sum_{j=1}^{p} \Xi_{j} z^{-j} \\
\sum_{j=1}^{p} \Lambda_{j} z^{-j} & I
\end{array}\right),
$$

where the $\Xi_{j}$ and $\Lambda_{j}$ are constant matrices. Such a transformation, which generalises 4.6), enables us to transform each $M_{r-j}^{(2)}$ in the same block diagonal form as $M_{r}^{(2)}$ with a convenient choice of $\Xi_{i}$ and $\Lambda_{i}$. Therefore, the initial system gives two decoupled sub-systems and, for each one, we proceed along the same lines as in the previous section. 
One can use this lemma to block diagonalise $M^{(2)}$, order by order : the two global blocks considered will be the nilpotent part of $M_{r}^{(2)}$ and its diagonalisable part. The latter can be dealt with using the procedure given in section IV B, while the former must be addressed using a generalised version of the procedure given in section IV C. We give here more details about the last part and, in the rest of this section, assume without loss of generality that $M_{r}^{(2)}$ contains only nilpotent blocks, such that

$$
M_{r}^{(2)}=\left(\begin{array}{ccccc}
J\left(n_{1}\right) & 0 & \cdots & & \\
0 & J\left(n_{2}\right) & 0 & \cdots & \\
\vdots & 0 & J\left(n_{3}\right) & 0 & \ldots \\
& \vdots & & & \ddots
\end{array}\right) \quad\left(\text { with } n_{1} \geq n_{2} \geq \cdots \geq n_{\text {last }}\right)
$$

The procedure in such a case requires to put the system in a specific normalized form. For a matrix $M$, obtained at a generic step in the algorithm, one says that the matrix is "normalized up to order $s$ " if all its leading terms $M_{r}, \cdots M_{r-s}$ have their $(K L)$ blocks verifying the following properties:

- either all rows are equal to zero except possibly the first one if $K \leq L$,

- or all columns are equal to zero except possibly the last one if $K>L$.

In order to reach this normalized form, one must use a succession of transformations ${ }^{12} P_{\text {norm }}(k)$ of the form

$$
P_{\text {norm }}(k)=I+\frac{1}{z^{k}} \Lambda,
$$

where $k$ varies from 1 to $s$. The matrix $\Lambda$ is a constant matrix, whose coefficients must be chosen, similarly to $\Xi$ in $(4.6)$, such that the new matrix $M$ is normalised, in the sense defined above $(\Lambda$ is uniquely defined if one requires that all its blocks $\Lambda^{K L}$ have zero last row if $K \leq L$ and zero first column if $K>L$ ). The procedure is iterative: if the system is normalized up to order $k$, it is possible to normalize it up to order $k+1$ by applying a transformation $P_{\text {norm }}(k+1)$. Indeed, this transformation will not modify any term of order higher than $r-k-1$.

The complete procedure to reduce the Poincaré rank of the matrix is then the following:

1. one starts with $s=1$;

2. one normalizes the system up to order $s$ using $P_{\text {norm }}(k)$ transformations ;

3. if $M_{r-s}$ is not block-diagonal, one uses a transformation $P_{u}(n)=\operatorname{diag}\left(I_{n_{1}}, I_{n_{2}}, \cdots, z^{s} I_{n_{\text {last }}}\right)$ and one goes back to step $1 ;^{13}$

4. if it is block-diagonal, one uses a $P_{p / q}$ transformation, which is a block form of 4.17 or 4.21:

$$
P_{p / q}=\left(\begin{array}{ccccc}
D\left(n_{1}, z^{p / q}\right) & 0 & \ldots & \\
0 & D\left(n_{2}, z^{p / q}\right) & 0 & \cdots & \\
\vdots & 0 & D\left(n_{3}, z^{p / q}\right) & 0 & \ldots \\
& \vdots & & & \ddots
\end{array}\right)
$$

\footnotetext{
${ }^{12}$ Let us emphasize on the fact that the hierarchy $n_{1} \geq n_{2} \geq \cdots$ is crucial for this step to succeed.

${ }^{13}$ It is proved in [19] that after a finite number of steps, one always gets a block-diagonal subleading term, which means that this procedure stops at some point and that one can go on with step 4 .
} 
where the matrices $D(n, z)$ have been defined in (4.17) and $p$ and $q$ are either co-prime integers (with $1 \leq p \leq q \leq n_{1}$ ) or equal in the case $p / q=1$;

5. if no $P_{p / q}$ transformation is admissible (see the definition after 4.21), one goes back to step 1 with $s$ increased by one. Otherwise, one stops here.

Thanks to the above procedure, one obtains either a system depending on $z$ with a reduced Poincaré rank, or a new system depending on $z^{p / q}$ with a non-nilpotent leading term. In the former case, one can simply pursue with the algorithm. In the latter case, one can change variables by writing $w=z^{p / q}$ and start the algorithm again.

\section{F. A higher dimensional example with $p / q \neq 1$}

We now present a higher dimensional $(n=5)$ example, adapted from [21], where the dominant term in the asymptotic expansion of the matrix $M$ has a non trivial canonical Jordan form with two Jordan blocks. The matrix $M(z)$ is given by

$$
M(z)=\left(\begin{array}{ccccc}
0 & z^{3} & -z & 1 & 2 z \\
-z^{2} & z & 0 & -z & 0 \\
z & 1 & 0 & z^{3} & 1 \\
1 & -z & 1 & z & z^{3} \\
z & 0 & -3 z & 0 & -1
\end{array}\right) \equiv M_{3} z^{3}+M_{2} z^{2}+M_{1} z+M_{0}
$$

where the leading term $M_{3}$ is nilpotent and has a 2-block Jordan structure.

We perform a first transformation $Y=P_{(1)} Y^{(1)}$ so that the leading term has now the following Jordan (lower triangular) canonical form (the matrix $P_{(1)}$ can easily been deduced):

$$
M^{(1)}(z)=\left(\begin{array}{ccccc}
-1 & 0 & -3 z & 0 & z \\
z^{3} & z & 1 & -z & 1 \\
1 & z^{3} & 0 & 1 & z \\
0 & -z & 0 & z & -z^{2} \\
2 z & 1 & -z & z^{3} & 0
\end{array}\right) \quad \Longrightarrow \quad M_{3}^{(1)}=\left(\begin{array}{ccccc}
0 & 0 & 0 & 0 & 0 \\
1 & 0 & 0 & 0 & 0 \\
0 & 1 & 0 & 0 & 0 \\
0 & 0 & 0 & 0 & 0 \\
0 & 0 & 0 & 1 & 0
\end{array}\right)
$$

The block structure of $M_{3}^{(1)}$ defines the layout that we will be using to compute the asymptotic expansion of the solution.

We notice that the next-to-leading term $M_{2}^{(1)}$ in the expansion of $M^{(1)}$ is already normalised. Therefore, we can immediately try to reduce the order of the system thanks to a new transformation $Y^{(1)}=P_{(2)} Y^{(2)}$,

$$
P_{(2)}=\left(\begin{array}{ccccc}
1 & 0 & 0 & 0 & 0 \\
0 & z & 0 & 0 & 0 \\
0 & 0 & z^{2} & 0 & 0 \\
0 & 0 & 0 & 1 & 0 \\
0 & 0 & 0 & 0 & z
\end{array}\right) \quad \Longrightarrow \quad M^{(2)}=\left(\begin{array}{ccccc}
-1 & 0 & -3 z^{3} & 0 & z^{2} \\
z^{2} & z-\frac{1}{z} & z & -1 & 1 \\
\frac{1}{z^{2}} & z^{2} & -\frac{2}{z} & \frac{1}{z^{2}} & 1 \\
0 & -z^{2} & 0 & z & -z^{3} \\
2 & 1 & -z^{2} & z^{2} & -\frac{1}{z}
\end{array}\right)
$$

However, we immediately see that the order of the system has not diminished. This example falls in the cases where we need to change the variable $z$ or, equivalently, i.e. to make a transformation of the form 4.21 for each Jordan block, We must therefore cancel the previous transformation 
(4.42) and instead consider $Y^{(1)}=\tilde{P}_{(2)} \tilde{Y}^{(2)}$, with

$$
\tilde{P}_{(2)}(z)=\left(\begin{array}{ccccc}
1 & 0 & 0 & 0 & 0 \\
0 & z^{p / q} & 0 & 0 & 0 \\
0 & 0 & z^{2 p / q} & 0 & 0 \\
0 & 0 & 0 & 1 & 0 \\
0 & 0 & 0 & 0 & z^{p / q}
\end{array}\right) .
$$

Following the method described below Eq. 4.21), we note that the largest Jordan block is of dimension 3, therefore we should take 2 co-prime integers between 1 and 3 for $p$ and $q$ with $p \leq q$. The possible choices for the ratio $p / q$ belong to the set $\{1 / 3,1 / 2,2 / 3\}$, since $p / q=1$ does not work. The largest value is $p / q=2 / 3$, which gives for the matrix $M^{(3)}$ the expression

$$
\left(\begin{array}{ccccc}
-1 & 0 & -3 z^{7 / 3} & 0 & z^{5 / 3} \\
z^{7 / 3} & z-\frac{2}{3 z} & z^{2 / 3} & -z^{1 / 3} & 1 \\
\frac{1}{z^{4 / 3}} & z^{7 / 3} & -\frac{4}{3 z} & \frac{1}{z^{4 / 3}} & z^{1 / 3} \\
0 & -z^{5 / 3} & 0 & z & -z^{8 / 3} \\
2 z^{1 / 3} & 1 & -z^{5 / 3} & z^{7 / 3} & -\frac{2}{3 z}
\end{array}\right)
$$

We observe that the subdiagonal terms have order $7 / 3$. To keep this value of $p / q$, we must make sure that no other term behaves like $z^{\alpha}$ with $\alpha>7 / 3$. However in this case there is a $z^{8 / 3}$ term. Therefore, the value $2 / 3$ is not admissible and we have to consider the next possible choice which is $p / q=1 / 2$. Such a change of variable leads to the matrix

$$
\tilde{M}^{(2)}=\left(\begin{array}{ccccc}
-1 & 0 & -3 z^{2} & 0 & z^{3 / 2} \\
z^{5 / 2} & z-\frac{1}{2 z} & \sqrt{z} & -\sqrt{z} & 1 \\
\frac{1}{z} & z^{5 / 2} & -\frac{1}{z} & \frac{1}{z} & \sqrt{z} \\
0 & -z^{3 / 2} & 0 & z & -z^{5 / 2} \\
2 \sqrt{z} & 1 & -z^{3 / 2} & z^{5 / 2} & -\frac{1}{2 z}
\end{array}\right) .
$$

Now, it verifies the requirements and we thus keep the value $p / q=1 / 2$ and continue the process.

The previous change of variable leads to a differential system where the coefficients of $M^{(3)}$ are non-integer powers functions of $z$. To apply the algorithm, we have to make a change of coordinate so that the system involves only integer powers of $z$. This can easily be done by introducing the new coordinate $u$ defined by $z=u^{2}$. As a consequence, the new differential system is now given by

$$
\frac{\mathrm{d} Y^{(3)}}{\mathrm{d} u}=M^{(3)}(u) Y^{(3)}, \quad M^{(3)}(u)=\left(\begin{array}{ccccc}
-2 u & 0 & -6 u^{5} & 0 & 2 u^{4} \\
2 u^{6} & \underline{2 u^{4}-1} & 2 u^{2} & -2 u^{2} & 2 u \\
\frac{2}{u} & 2 u^{6} & -\frac{2}{u} & \frac{2}{u} & 2 u^{2} \\
0 & -2 u^{4} & 0 & 2 u^{3} & -2 u^{6} \\
4 u^{2} & 2 u & -2 u^{4} & 2 u^{6} & -\frac{1}{u}
\end{array}\right),
$$

where $Y^{(3)}(u) \equiv \tilde{Y}^{(2)}(z)$ and $M^{(3)}(u) \equiv 2 u \tilde{M}^{(2)}(z)$ with $z=u^{2}$. As the leading term is not nilpotent, we keep the value of $p / q$. If it had been nilpotent, we would have had to go back one step and normalise up to the next order.

We can continue the algorithm with this new system: we will to do a new change of variables, reduce the order, and decouple the system... We will not present more steps as the rest of the computations is similar to what was done here and in previous sections. Nonetheless, for the sake 
of completeness, we give the final result. We show that, after enough steps of the algorithm, the initial system can be equivalently reformulated as

$$
\frac{\mathrm{d} Y^{(4)}}{\mathrm{d} w}=M^{(4)}(w) Y^{(4)}
$$

where $w=z^{1 / 6}$ and $M^{(4)}(w)$ is the following diagonal matrix

$$
\begin{aligned}
M^{(4)}(w)=\operatorname{Diag}\left[3^{4 / 3}(1-i \sqrt{3}) w^{19}+2 w^{11},-2 \times 3^{4 / 3} w^{19}+2 w^{11},\right. \\
\left.3^{4 / 3}(1+i \sqrt{3}) w^{19}+2 w^{11}, 6 i w^{20}+3 w^{11},-6 i w^{20}+3 w^{11}\right]+\mathcal{O}\left(w^{9}\right),
\end{aligned}
$$

up to order $\mathcal{O}\left(w^{9}\right)$. Integrating such a system is immediate and yields the leading orders of the asymptotic expansion of $Y^{(4)}$ from which we can extract the asymptotic expansion of the original variable $Y$.

\section{CONCLUSION}

In this work, we have studied the asymptotic behaviours, both at spatial infinity and near the horizon, of the linear perturbations about Schwarzschild black holes. Instead of following the traditional approach that consists in rewriting the equations of motion in the form of a stationary Schrödinger-like equation, which is second-order with respect to the radial coordinate, we have worked directly with the first-order equations of motion (in the frequency domain). For this direct approach to the asymptotic behaviour, we have used an algorithm that has been developed in several recent articles published in mathematical journals.

The principle of this algorithm is to transform the differential system, via successive changes of functions, until it can be written in an explicitly diagonal form, up to the required order (in the small parameter characterising the asymptotic regime). This procedure automatically provides the combination of the metric perturbations that encapsulates the physical degree of freedom in this asymptotic region and enables one to separate the ingoing and outgoing physical modes. Although we have worked in the standard Regge-Wheeler gauge, the same approach would work similarly for any other gauge choice.

Beyond its application to the perturbations of black holes, this systematic approach to the asymptotic behaviour could be very useful for similar problems in other domains of physics. This is why we have devoted the last part of this paper to a pedagogical presentation of the algorithm, with a few illustrative examples.

For black holes, the knowledge of the asymptotic behaviour of the perturbations is an indispensable first step in the determination of the quasi-normal modes. Indeed, these modes are characterised by the following boundary conditions: a purely outgoing behaviour at spatial infinity and purely ingoing behaviour at the horizon. Imposing these boundary conditions, we have shown that the known quasi-normal modes can be recovered numerically, without resorting to the Schrödinger-like formulation, thus providing an alternative approach to the standard method. We stress that our rudimentary numerical calculation was simply to illustrate the feasibility of this new approach, without trying to reach the precision and efficiency of the powerful numerical methods that have been developed in the traditional approach.

This novel approach could be especially useful in the context of generalised black hole solutions, for instance in modified gravity theories, where the equations of motion for the perturbations are different and extra fields can be present. In a companion paper, we have applied the same algorithm to a few black holes solutions within scalar-tensor theories that belong to the most general known family: DHOST (Degenerate Higher-Order Scalar-Tensor) theories. The same method could be applied to the study of other types of black holes, or even completely different physical systems. 
As a final remark, let us stress that this approach could be used to get some analytical insight on the asymptotic behaviour of the modes by looking directly at the structure of the matrix coefficients that are relevant. In this sense, it might provide a pre-diagnosis tool to explore the healthiness of some black hole solutions without resorting to a full numerical investigation.

\section{Acknowledgments}

We would like to thank Oleg Lisovyi for instructive discussions and guidance on the mathematics literature, as well as Emanuele Berti and Vitor Cardoso for very useful correspondence on quasinormal modes. We also thank Leo Stein for pointing out a case which was not covered in our previous version. We have used Mathematica for many of the calculations involved in this work. KN acknowledges the support from the CNRS grant 80PRIME and thanks the Laboratory of Physics at the ENS in Paris for its hospitality.

\section{Appendix A: Gauge transformations}

For completeness, we summarise in this Appendix the gauge fixing procedure for polar and axial perturbations about a Schwarzschild black hole in General Relativity, as originally discussed in [3] and [4].

Due to the invariance of the theory under space-time diffeomorphisms, the metric perturbations are not completely determined $h_{\mu \nu}$. Indeed, any infinitesimal change of coordinates $x^{\mu} \rightarrow x^{\mu}+\xi^{\mu}$ induces the transformation

$$
h_{\mu \nu} \rightarrow h_{\mu \nu}+\nabla_{\mu} \xi_{\nu}+\nabla_{\nu} \xi_{\mu}
$$

at the linear level. These transformations can be "projected" in the axial or polar sectors, which we examine in turn.

\section{Axial perturbations: Regge-Wheeler gauge}

Before gauge fixing, axial perturbations are parametrised by three families of functions $h_{0}^{\ell m}$, $h_{1}^{\ell m}$ and $h_{2}^{\ell m}$ of the variables $(r, t)$, according to

$$
\begin{aligned}
& h_{t \theta}=\frac{1}{\sin \theta} \sum_{\ell, m} h_{0}^{\ell m}(t, r) \partial_{\varphi} Y_{\ell m}(\theta, \varphi), \quad h_{t \varphi}=-\sin \theta \sum_{\ell, m} h_{0}^{\ell m}(t, r) \partial_{\theta} Y_{\ell m}(\theta, \varphi), \\
& h_{r \theta}=\frac{1}{\sin \theta} \sum_{\ell, m} h_{1}^{\ell m}(t, r) \partial_{\varphi} Y_{\ell m}(\theta, \varphi), \quad h_{r \varphi}=-\sin \theta \sum_{\ell, m} h_{1}^{\ell m}(t, r) \partial_{\theta} Y_{\ell m}(\theta, \varphi), \\
& h_{a b}=\sin \theta \sum_{\ell, m} h_{2}^{\ell m}(t, r) \epsilon_{c(a} D^{c} \partial_{b)} Y_{\ell m}(\theta, \varphi),
\end{aligned}
$$

where, in the last equation, the indices $a$ and $b$ belong to the set $\{\theta, \varphi\}, \epsilon_{a b}$ is the totally antisymmetric symbol such that $\epsilon_{\theta \varphi}=+1$ and $D_{a}$ is the 2-dimensional covariant derivative associated with the metric of the 2 -sphere $d \theta^{2}+\sin ^{2} \theta d \varphi^{2}$. More explicitely, the angular components of the 
metric can be written

$$
\begin{aligned}
& h_{\theta \theta}=\sum_{\ell, m} \frac{1}{\sin \theta} h_{2}^{\ell m}(t, r)\left(\partial_{\theta} \partial_{\varphi}-\operatorname{cotan} \theta \partial_{\varphi}\right) Y_{\ell m}(\theta, \varphi), \\
& h_{\theta \varphi}=h_{\varphi \theta}=-\sum_{\ell, m} \sin \theta h_{2}^{\ell m}(t, r)\left(\frac{\ell(\ell+1)}{2}+\partial_{\theta}^{2}\right) Y_{\ell m}(\theta, \varphi), \\
& h_{\varphi \varphi}=-\sum_{\ell, m} h_{2}^{\ell m}(t, r) \sin \theta\left(\partial_{\theta} \partial_{\varphi}-\operatorname{cotan} \theta \partial_{\varphi}\right) Y_{\ell m}(\theta, \varphi) .
\end{aligned}
$$

All the other components of the axial perturbations vanish.

In the axial sector, the nonzero components of the generator $\xi^{\mu}$ that preserves the odd parity of the perturbations can be decomposed into spherical harmonics as follows,

$$
\xi_{\theta}=\sum_{\ell, m} \xi^{\ell m}(t, r) \partial_{\theta} Y_{\ell, m}(\theta, \varphi), \quad \xi_{\varphi}=\sum_{\ell, m} \xi^{\ell m}(t, r) \partial_{\varphi} Y_{\ell, m}(\theta, \varphi),
$$

and the induced gauge transformations on the functions $h_{0}, h_{1}$ and $h_{2}$ are given, according to (A1), by

$$
h_{0} \rightarrow h_{0}-\dot{\xi}, \quad h_{1} \rightarrow h_{1}-\xi^{\prime}+\frac{2}{r} \xi, \quad h_{2} \rightarrow h_{2}-2 \xi,
$$

where we have dropped the indices $(\ell m)$ for simplicity. A dot and a prime denote a derivative with respect to $t$ and $r$, respectively.

As a consequence, one can always choose a gauge in which $h_{2}^{\ell m}=0$ which is the well-known Regge-Wheeler gauge for the axial perturbations [3]. Notice that this gauge choice is possible for $\ell \geq 2$ only (the cases $\ell=0$ and $\ell=1$ will be discussed later below).

\section{Even-parity or polar perturbations: Zerilli gauge}

Before gauge fixing, polar perturbations of the metric are parametrised by seven families of functions $H_{0}^{\ell m}, H_{1}^{\ell m}, H_{2}^{\ell m}, \alpha^{\ell m}, \beta^{\ell m}, K^{\ell m}$ and $G^{\ell m}$ of the variables $(r, t)$ which appear in the components of the metric perturbations as follows,

$$
\begin{aligned}
h_{t t} & =A(r) \sum_{\ell, m} H_{0}^{\ell m}(t, r) Y_{\ell m}(\theta, \varphi), \quad h_{t r}=\sum_{\ell, m} H_{1}^{\ell m}(t, r) Y_{\ell m}(\theta, \varphi), \\
h_{r r} & =\frac{1}{A(r)} \sum_{\ell, m} H_{2}^{\ell m}(t, r) Y_{\ell m}(\theta, \varphi), \\
h_{t a} & =\sum_{\ell, m} \beta^{\ell m}(t, r) \partial_{a} Y_{\ell m}(\theta, \varphi), \quad h_{r a}=\sum_{\ell, m} \alpha^{\ell m}(t, r) \partial_{a} Y_{\ell m}(\theta, \varphi), \\
h_{a b} & =\sum_{\ell, m} K^{\ell m}(t, r) g_{a b} Y_{\ell m}(\theta, \varphi)+\sum_{\ell, m} G^{\ell m}(t, r) D_{a} D_{b} Y_{\ell m}(\theta, \varphi) .
\end{aligned}
$$

More precisely, the angular part of the metric can be written as

$$
\begin{aligned}
h_{\theta \theta} & =\sum_{\ell, m} K^{\ell m}(t, r) Y_{\ell m}(\theta, \varphi)+\sum_{\ell, m} G^{\ell m}(t, r) \partial_{\theta}^{2} Y_{\ell m}(\theta, \varphi), \\
h_{\theta \varphi} & =h_{\varphi \theta}=-\sum_{\ell, m} G^{\ell m}(t, r) \operatorname{cotan} \theta \partial_{\varphi} Y_{\ell m}(\theta, \varphi), \\
h_{\varphi \varphi} & =\sum_{\ell, m} \sin ^{2} \theta K^{\ell m}(t, r) Y_{\ell m}(\theta, \varphi)+\sum_{\ell, m} G^{\ell m}(t, r)\left(\partial_{\varphi}^{2}+\sin \theta \cos \theta \partial_{\theta}\right) Y_{\ell m}(\theta, \varphi) .
\end{aligned}
$$


Similarly to the axial sector, this parametrisation is redundant and can be simplified by gauge fixing. Now, linear diffeomorphisms which preserve even-parity of the metric components are generated by vector fields $\xi$ whose components decompose into spherical harmonics as follows,

$$
\begin{aligned}
& \xi_{t}=\sum_{\ell, m} T^{\ell m}(t, r) Y_{\ell m}(\theta, \varphi), \quad \xi_{r}=\sum_{\ell, m} R^{\ell m}(t, r) Y_{\ell m}(\theta, \varphi), \\
& \xi_{\theta}=\sum_{\ell, m} \Theta^{\ell m}(t, r) \partial_{\theta} Y_{\ell m}(\theta, \varphi), \quad \xi_{\varphi}=\sum_{\ell, m} \Theta^{\ell m}(t, r) \partial_{\varphi} Y_{\ell m}(\theta, \varphi) .
\end{aligned}
$$

Here $T^{\ell m}, R^{\ell m}$ and $\Theta^{\ell m}$ are arbitrary functions of $(t, r)$. These linear diffeomorphisms induce gauge transformations on the functions that parametrise metric perturbations according to

$$
\begin{aligned}
& H_{0}^{\ell m}(t, r) \longrightarrow H_{0}^{\ell m}(t, r)+\frac{2}{A(r)} \dot{T}^{\ell m}(t, r)+A^{\prime}(r) R^{\ell m}(t, r), \\
& H_{1}^{\ell m}(t, r) \longrightarrow H_{1}^{\ell m}(t, r)+\dot{R}^{\ell m}(t, r)+T^{\ell m}(t, r)+\frac{A^{\prime}(r)}{A(r)} T^{\ell m}(t, r), \\
& H_{2}^{\ell m}(t, r) \longrightarrow H_{2}^{\ell m}(t, r)+2 A(r) R^{\ell l m}(t, r)-A^{\prime}(r) R_{\ell m}(t, r), \\
& \beta^{\ell m}(t, r) \longrightarrow \beta^{\ell m}(t, r)+T^{\ell m}(t, r)+\dot{\Theta}^{\ell m}(t, r), \\
& \alpha^{\ell m}(t, r) \longrightarrow \alpha^{\ell m}(t, r)+R^{\ell m}(t, r)+\Theta^{\ell \ell m}(t, r)-\frac{2}{r} \Theta^{\ell m}(t, r), \\
& K^{\ell m}(t, r) \longrightarrow K^{\ell m}(t, r)+\frac{2 A(r)}{r} R^{\ell m}(t, r), \\
& G^{\ell m}(t, r) \longrightarrow G^{\ell m}(t, r)+2 \Theta^{\ell m}(t, r) .
\end{aligned}
$$

An immediate consequence of the gauge transformations is that one can choose the gauge parameter $\xi$ such that $G^{\ell m}=0$ by fixing $\Theta^{\ell m}$, then $\alpha^{\ell m}=0$ and $\beta^{\ell m}=0$ by fixing $R^{\ell m}$ and $T^{\ell m}$ respectively, in the case where $\ell \geq 2$. This gauge is known as the Zerilli gauge [4] (see [26] for a recent presentation in the context of modified gravity).

\section{Monopole and dipole perturbations}

We consider here the special cases $\ell=0$ and $\ell=1$.

\section{a. Axial modes}

For the axial modes, the components $h_{a b}$ vanish identically for $\ell=1$ (axial perturbations do not have $\ell=0$ components) which means that $h_{2}$ does not show up in the components of the metric. Hence, when $\ell=1$, it is necessary to make a different gauge choice. In general, one chooses $h_{1}=0$ which fixes the gauge parameter $\xi$ up to a function of the form $C(t) r^{2}$. Therefore, $h_{0}$ inherits a residual gauge invariance given by $h_{0} \rightarrow h_{0}+F(t) r^{2}$ where $F(t)$ is an arbitrary function. Then $h_{0}$ can be shown to satisfy the equation of motion,

$$
2 h_{0}(r)-r h_{0}^{\prime}(r)=0 .
$$

Therefore, the mode $h_{0}$ is not propagating. 


\section{b. Polar modes}

Let us now turn to polar perturbations. In the case $\ell=0, H_{0}, H_{1}, H_{2}$ and $K$ are the only non-vanishing components of the metric perturbations whereas $T$ and $R$ are the only non-vanishing components of the gauge parameter (so that the gauge transformation preserves the monopole). As in the general case, one can choose $R$ to fix $K=0$. Then, one can in principle make use of $T$ to get rid of $H_{1}$ (we could have also set $H_{0}=0$ ). Finally, we are left with only two non-vanishing functions which are either $H_{2}$ or $H_{0}$ and we will compute the corresponding equations of motion in the next section.

The main difference, concerning the gauge fixing, between the general case and the case $\ell=1$ lies in the fact that, in the latter, $h_{a b}$ can be shown to depend on the difference $G-K$ only, so that one can fix $K=0$ without loss of generality. Furthermore, one can make the gauge fixing $G=0$ by an appropriate choice of $\Theta$. Then, one makes use of $T$ to fix $\beta=0$. Finally, one uses the remaining free gauge function $R$ to fix $\alpha=0$. At the end, we are left with the three non-vanishing functions $H_{0}, H_{1}$ and $H_{2}$. The dynamics of these three free parameters will be studied in the next section as well.

Concerning the monopole $(\ell=0)$, we showed in section II A 3 that its dynamics is fully described in terms of the functions $H_{0}$ and $H_{2}$ only, as all the others can be sent to 0 by gauge fixing. Thus the equations of motion simplifies drastically and, after some calculations, give

$$
H_{0}(r)-H_{2}(r)=0, \quad H_{2}(r)+\left(r-r_{s}\right) H_{2}^{\prime}(r)=0 .
$$

The solution reads $H_{2}(r)=C /\left(r-r_{s}\right)$ and the mode is not propagating.

Finally, the dynamics of the polar dipole $(\ell=1)$ is described by the three non-vanishing functions $H_{0}, H_{1}$ and $H_{2}$ which satisfy the three independent equations,

$$
\begin{aligned}
& 2 H_{2}(r)+\left(r-r_{s}\right) H_{2}^{\prime}(r)=0, \quad H_{1}(r)+i \omega H_{2}(r)=0, \\
& H_{0}(r)+\left(r_{s}-r\right) H_{0}^{\prime}(r)-2 i r \omega H_{1}(r)+H_{2}(r)=0 .
\end{aligned}
$$

Indeed, the full set of the original Einstein equations is equivalent to this one which can easily be solved explicitly but its solution is not relevant for our purpose. Nonetheless, we see immediately from the equations that, like the monopole, the polar dipole does not propagate. This is why we do not consider it in the rest of the paper.

\section{Appendix B: Equations of motion for the polar perturbations}

In this appendix, we present the equations of motion satisfied by the polar perturbations and show how the system (2.14) is obtained. The Euler-Lagrange equations equations of motion (2.4) 
yield, in the polar sector,

$$
\begin{aligned}
\mathcal{E}_{t t}= & -2(\lambda+2)\left(1-\frac{r_{s}}{r}\right) H_{2}(t, r)-2 \lambda\left(1-\frac{r_{s}}{r}\right) K(t, r)-\frac{2}{r}\left(r-r_{s}\right)^{2} \frac{\partial H_{2}}{\partial r} \\
& +\left(6 r-11 r_{s}+\frac{5 r_{s}^{2}}{r}\right) \frac{\partial K}{\partial r}+2\left(r-r_{s}\right)^{2} \frac{\partial^{2} K}{\partial r^{2}}=0, \\
\mathcal{E}_{t r}= & -2(\lambda+1) H_{1}(t, r)-2 r \frac{\partial H_{2}}{\partial t}+r \frac{2 r-3 r_{s}}{r-r_{s}} \frac{\partial K}{\partial t}+2 r^{2} \frac{\partial^{2} K}{\partial t \partial r}=0, \\
\mathcal{E}_{r r}= & -2 \frac{\lambda+1}{1-r_{s} / r} H_{0}(t, r)+\frac{2}{1-r_{s} / r} H_{2}(t, r)+\frac{2 \lambda}{1-r_{s} / r} K(t, r)+2 r \frac{\partial H_{0}}{\partial r}-r \frac{2 r-r_{s}}{2\left(r-r_{s}\right)} \frac{\partial K}{\partial r} \\
& -\frac{4 r^{2}}{r-r_{s}} \frac{\partial H_{1}}{\partial t}+\frac{2 r^{4}}{\left(r-r_{s}\right)^{2}} \frac{\partial^{2} K}{\partial t^{2}}=0, \\
\mathcal{E}_{t \theta}= & -\frac{r_{s}}{r} H_{1}(t, r)-\left(r-r_{s}\right) \frac{\partial H_{1}}{\partial r}+r \frac{\partial H_{2}}{\partial t}+r \frac{\partial K}{\partial t}=0, \\
\mathcal{E}_{r \theta}= & \frac{2 r-3 r_{s}}{2\left(r-r_{s}\right)} H_{0}(t, r)-\frac{2 r-r_{s}}{2\left(r-r_{s}\right)} H_{2}(t, r)-r \frac{\partial H_{0}}{\partial r}+r \frac{\partial K}{\partial r}+\frac{r^{2}}{r-r_{s}} \frac{\partial H_{1}}{\partial t}=0, \\
\mathcal{E}_{\theta \theta}= & \frac{2 r+r_{s}}{2} \frac{\partial H_{0}}{\partial r}+\frac{2 r-r_{s}}{2} \frac{\partial H_{2}}{\partial r}-\left(2 r-r_{s}\right) \frac{\partial K}{\partial r}+r\left(r-r_{s}\right) \frac{\partial^{2} H_{0}}{\partial r^{2}}-r\left(r-r_{s}\right) \frac{\partial^{2} K}{\partial r^{2}} \\
& -r \frac{2 r-r_{s}}{r-r_{s}} \frac{\partial H_{1}}{\partial t}-2 r^{2} \frac{\partial^{2} H_{1}}{\partial t \partial r}+\frac{r^{3}}{r-r_{s}} \frac{\partial^{2} H_{2}}{\partial t^{2}}+\frac{r^{3}}{r-r_{s}} \frac{\partial^{2} K}{\partial t^{2}}=0, \\
\mathcal{E}_{\theta \varphi}= & H_{0}(t, r)-H_{2}(t, r)=0
\end{aligned}
$$

The equations of motion $\mathcal{E}_{t \varphi}=0, \mathcal{E}_{r \varphi}=0$ and $\mathcal{E}_{\varphi \varphi}=0$ are identical to $\mathcal{E}_{t \theta}=0, \mathcal{E}_{r \theta}=0$ and $\mathcal{E}_{\theta \theta}=0$, respectively.

We can immediately solve the last equation of the system (B1) and replace $H_{2}$ by $H_{0}$ in all the other equations. We thus get six equations for only three independent functions $K, H_{0}$ and $H_{1}$, and we want to extract three "simple" independent equations out of them. One can then note that the combination

$$
\mathcal{E} \equiv \frac{i r_{s}}{4 \omega r\left(r-r_{s}\right)} \mathcal{E}_{t r}+\frac{1}{2} \mathcal{E}_{r r}+\mathcal{E}_{r \theta}
$$

is purely algebraic, i.e. it does not involve any derivatives of the functions. Moreover, we find that the system $\mathcal{E}_{t r}, \mathcal{E}_{t \theta}, \mathcal{E}_{r \theta}, \mathcal{E}$ enables us to recover $\mathcal{E}_{t t}$ and $\mathcal{E}_{\theta \theta}$ so that we can restrict immediately to the system formed by these four equations which, after some simple calculations, are given by the system of differential equations

$$
\begin{aligned}
& K^{\prime}(r)-\frac{1}{r} H_{0}(r)-\frac{i(\lambda+1)}{\omega r^{2}} H_{1}(r)+\frac{1}{r} \frac{2 r-3 r_{s}}{2\left(r-r_{s}\right)} K(r)=0, \\
& H_{1}^{\prime}(r)+\frac{i \omega r}{r-r_{s}} H_{0}(r)+\frac{r_{s}}{r\left(r-r_{s}\right)} H_{1}(r)+\frac{i \omega r}{r-r_{s}} K(r)=0, \\
& H_{0}^{\prime}(r)-K^{\prime}(r)+\frac{r_{s}}{r\left(r-r_{s}\right)} H_{0}(r)+\frac{i \omega r}{r-r_{s}} H_{1}(r)=0,
\end{aligned}
$$

together with the algebraic equation

$$
\left(\frac{3 r_{s}}{r}+2 \lambda\right) H_{0}(r)+\left(\frac{i r_{s}(\lambda+1)}{\omega r^{2}}-2 i \omega r\right) H_{1}(r)+\frac{3 r_{s}^{2}+2 r_{s}(2 \lambda-1) r-4 \lambda r^{2}+4 \omega^{2} r^{4}}{2 r\left(r-r_{s}\right)} K(r)=0 .
$$

One equation is still redundant. However, we can solve the algebraic equation for $H_{0}$ and substitute its expression into the first three equations. This shows that the third is not independent from the 
first two. Finally, we obtain

$$
\begin{aligned}
& K^{\prime}(r)=\frac{3 r_{s}^{2}+r_{s}(\lambda-2) r-2 \omega^{2} r^{4}}{r\left(r-r_{s}\right)\left(3 r_{s}+2 \lambda r\right)} K(r)+\frac{i}{\omega r^{2}}\left(\lambda+1+\frac{-r_{s}(\lambda+1)+2 \omega^{2} r^{3}}{3 r_{s}+2 \lambda r}\right) H_{1}(r), \\
& H_{1}^{\prime}(r)=\frac{i r\left(9 r_{s}^{2}+8 r_{s}(\lambda-1) r-8 \lambda r^{2}+4 \omega^{2} r^{4}\right)}{2\left(r-r_{s}\right)^{2}\left(3 r_{s}+2 \lambda r\right)} \omega K(r)-\frac{3 r_{s}^{2}+r_{s}(1+3 \lambda) r-2 \omega^{2} r^{4}}{r\left(r-r_{s}\right)\left(3 r_{s}+2 \lambda r\right)} H_{1}(r),
\end{aligned}
$$

and we obtain the required form (2.14) with the definitions $X_{1}(r) \equiv K(r)$ and $X_{2}(r) \equiv H_{1}(r) / \omega$.

\section{Appendix C: Flowchart for the algorithm}

In this appendix, we draw a flowchart to illustrate the algorithm that we are using to compute the asymptotic behaviour of a solution of a first order system.

It should be noted that, in principle, one can skip the first question "Is the leading term diagonalisable?" and put directly the leading order term in its Jordan form. Indeed, when the leading term is diagonalisable, putting it into its Jordan form is equivalent to diagonalising it and the resulting Jordan matrix is made of $d$ one-dimensional blocks where $d$ is the dimension of the system, thus of the matrix. Therefore, the procedure for splitting the system into several subsystems described in section IVE is in this case equivalent to the procedure described in section IV B where we are treating several blocks. 


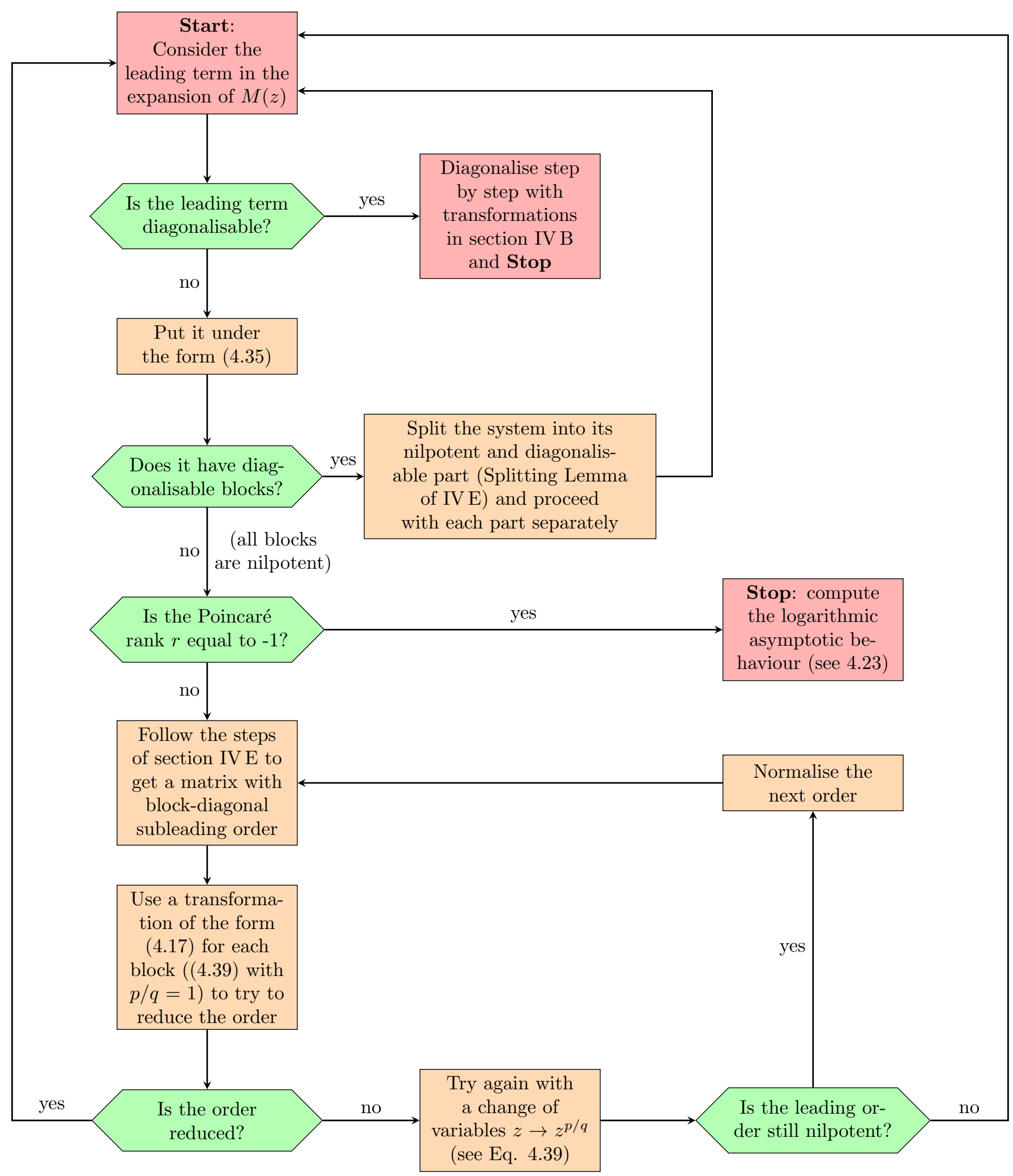


[1] E. Berti, V. Cardoso, and C. M. Will, "On gravitational-wave spectroscopy of massive black holes with the space interferometer LISA," Phys. Rev. D 73 (2006) 064030, gr-qc/0512160.

[2] E. Berti, K. Yagi, H. Yang, and N. Yunes, "Extreme Gravity Tests with Gravitational Waves from Compact Binary Coalescences: (II) Ringdown," Gen. Rel. Grav. 50 (2018), no. 5 49, 1801.03587.

[3] T. Regge and J. A. Wheeler, "Stability of a Schwarzschild singularity," Phys. Rev. 108 (1957) 1063-1069.

[4] F. J. Zerilli, "Effective potential for even parity Regge-Wheeler gravitational perturbation equations," Phys. Rev. Lett. 24 (1970) 737-738.

[5] R. McManus, E. Berti, C. F. B. Macedo, M. Kimura, A. Maselli, and V. Cardoso, "Parametrized black hole quasinormal ringdown. II. Coupled equations and quadratic corrections for nonrotating black holes," Phys. Rev. D 100 (2019), no. 4 044061, 1906.05155.

[6] D. Langlois, K. Noui, and H. Roussille, "Black hole perturbations in modified gravity," 2103.14750

[7] D. Langlois and K. Noui, "Degenerate higher derivative theories beyond Horndeski: evading the Ostrogradski instability," JCAP 1602 (2016), no. 02 034, 1510.06930.

[8] M. Crisostomi, K. Koyama, and G. Tasinato, "Extended Scalar-Tensor Theories of Gravity," JCAP 1604 (2016), no. $04044,1602.03119$.

[9] J. Ben Achour, D. Langlois, and K. Noui, "Degenerate higher order scalar-tensor theories beyond Horndeski and disformal transformations," Phys. Rev. D93 (2016), no. 12 124005, 1602.08398.

[10] J. Ben Achour, M. Crisostomi, K. Koyama, D. Langlois, K. Noui, and G. Tasinato, "Degenerate higher order scalar-tensor theories beyond Horndeski up to cubic order," JHEP 12 (2016) 100, 1608.08135 .

[11] K. Takahashi and H. Motohashi, "Black hole perturbations in DHOST theories: Master variables, gradient instability, and strong coupling," JCAP 08 (2021) 013, 2106.07128.

[12] S. Chandrasekhar, "The mathematical theory of black holes," in Oxford, UK: Clarendon (1992) 646 p., Oxford, UK: Clarendon (1985) 646 P., 1985.

[13] E. W. Leaver, "An Analytic representation for the quasi normal modes of Kerr black holes," Proc. Roy. Soc. Lond. A402 (1985) 285-298.

[14] K. D. Kokkotas and B. G. Schmidt, "Quasinormal modes of stars and black holes," Living Rev. Rel. 2 (1999) 2, gr-qc/9909058.

[15] H.-P. Nollert, "Topical Review: Quasinormal modes: the characteristic 'sound' of black holes and neutron stars," Class. Quant. Grav. 16 (1999) R159-R216.

[16] E. Berti, V. Cardoso, and A. O. Starinets, "Quasinormal modes of black holes and black branes," Class. Quant. Grav. 26 (2009) 163001, 0905.2975

[17] R. A. Konoplya and A. Zhidenko, "Quasinormal modes of black holes: From astrophysics to string theory," Rev. Mod. Phys. 83 (2011) 793-836, 1102.4014.

[18] W. Wasow, Asymptotic Expansions for Ordinary Differential Equations. Courier Dover publications, 1965.

[19] W. Balser, "Computation of formal fundamental solutions," Linear Algebra and its Applications 288 (Feb., 1999) 293-312.

[20] M. Barkatou and E. Pflügel, "An Algorithm Computing the Regular Formal Solutions of a System of Linear Differential Equations," Journal of Symbolic Computation 28 (Oct., 1999) 569-587.

[21] E. Pflügel, "A Root-Free Splitting-Lemma for Systems of Linear Differential Equations," arXiv:1911.05837 [cs] (Nov., 2019). arXiv: 1911.05837.

[22] A. Jansen, "Overdamped modes in Schwarzschild-de Sitter and a Mathematica package for the numerical computation of quasinormal modes," Eur. Phys. J. Plus 132 (2017), no. 12 546, 1709.09178 .

[23] J. P. Boyd, Chebyshev \& Fourier Spectral Methods. Springer Berlin Heidelberg, 1989.

[24] M. A. Barkatou, "An algorithm to compute the exponential part of a formal fundamental matrix solution of a linear differential system," Applicable Algebra in Engineering, Communication and Computing 8 (Jan., 1997) 1-23.

[25] Abdelaziz Hilali, Solutions formelles de systèmes différentiels linéaires au voisinage d'un point singulier. PhD thesis, Université Joseph-Fourier - Grenoble I, 1987.

[26] T. Kobayashi, H. Motohashi, and T. Suyama, "Black hole perturbation in the most general 
scalar-tensor theory with second-order field equations II: the even-parity sector," Phys. Rev. D89 (2014), no. $8084042,1402.6740$. 